\title{
A Stress-Dependent Hysteresis Model for Ferroelectric Materials
}

\author{
Brian L. Ball ${ }^{1}$, Ralph C. Smith ${ }^{2}$, Sang-Joo Kim ${ }^{3}$ and Stefan Seelecke ${ }^{4}$ \\ 1,2 Center for Research in Scientific Computation \\ Department of Mathematics \\ North Carolina State University \\ Raleigh, NC 27695 \\ ${ }^{3}$ Mechanical and Information Engineering \\ University of Seoul \\ Seoul, 130-743 Korea \\ 4 Mechanical and Aerospace Engineering \\ North Carolina State University \\ Box 7910 \\ Raleigh, NC 27695
}

\begin{abstract}
This paper addresses the development of homogenized energy models which characterize the ferroelastic switching mechanisms inherent to ferroelectric materials in a manner suitable for subsequent transducer and control design. In the first step of the development, we construct Helmholtz and Gibbs energy relations which quantify the potential and electrostatic energy associated with $90^{\circ}$ and $180^{\circ}$ dipole orientations. Equilibrium relations appropriate for homogeneous materials in the absence or presence of thermal relaxation are respectively determined by minimizing the Gibbs energy or balancing the Gibbs and relative thermal energies using Boltzmann principles. In the final step of the development, stochastic homogenization techniques are employed to construct macroscopic models suitable for nonhomogeneous, polycrystalline compounds. Attributes and limitations of the characterization framework are illustrated through comparison with experimental PLZT data.
\end{abstract}

\footnotetext{
${ }^{1}$ Email: blball@unity.ncsu.edu, Telephone: (919) 332-7957

${ }^{2}$ Email: rsmith@eos.ncsu.edu, Telephone: (919) 515-7552

${ }^{3}$ Email: sjk@uos.ac.kr, Telephone: 82-2-2210-2757

${ }^{4}$ Email: stefan_seelecke@ncsu.edu, Telephone: (919) 515-5282
} 


\section{Introduction}

Transducers exploiting ferroelectric compounds - e.g., PZT, PLZT and PMN — offer unique actuator and sensor capabilities due to the electromechanical coupling inherent to the compounds. A fundamental attribute of these materials, when operating within the ferroelectric regimes typically considered for transducer design, is the presence of hysteresis and constitutive nonlinearities due to the noncentrosymmetric structure of the compounds. These nonlinearities can be reduced by regulating input fields or voltages, employing feedback designs, or utilizing charge or current-controlled amplifiers, in which case, linear constitutive relations provide reasonable accuracy. At higher drive levels, however, the hysteresis and nonlinearities associated with dipole switching must be accommodated in models, transducer designs, and control algorithms to achieve design specifications.

As detailed in Section 2, hysteresis and constitutive nonlinearities in ferroelectric materials can be attributed to both ferroelectric and ferroelastic switching. The former is typically due to field-induced $90^{\circ}$ and $180^{\circ}$ dipole switching whereas the latter is associated with stress-induced $90^{\circ}$ switching. Due to the electromechanical coupling inherent to compounds such as PZT, PLZT and PMN, both effects are manifested in transducers operating in high drive regimes.

To illustrate, consider the PZT-based THUNDER (Thin layered UNimorph Driver and sEnsoR) transducer shown in Figure 1(a). As detailed in [24], THUNDER transducers have been considered for applications ranging from high speed valve design to shape modification in an airfoil. Due to their construction, they are capable of achieving large displacements due to a combination of robustness provided by the metallic backing layer and curvature/stress enhancement achieved during fabrication. However, the prestresses also produce the asymmetric hysteresis behavior shown in Figure 1(b) which necessitates the use of models which incorporate $90^{\circ}$ ferroelastic switching.

Modeling hierarchies for quantifying ferroelastic switching can be characterized by the level at which energy or phenomenological principles are initiated since essentially all the frameworks results in macroscopic models or constitutive relations commensurate with experimental measurements or transducer design. Micromechanical models are typically associated with energy characterization or thermodynamic principles developed at the level of a single lattice cell, single domain, or single crystal. Subsequent macroscopic models are obtained through brute force computations or various homogenization techniques. Material characterization via large scale computations can be used to quantify fundamental mechanisms whereas the low-order homogenized models facilitate implementation. Additionally, the effective parameters resulting from homogenization can phenomenologically quantify unknown physics or model uncertainty, thus yielding models that are accurate for a range of operating conditions. Additional details regarding micromechanical models for materials can be found in the summaries $[10,12,24]$ and references $[11,16,17]$.

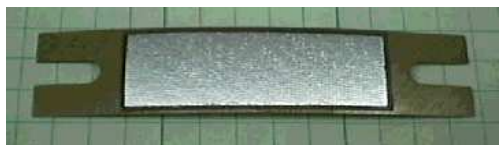

(a)

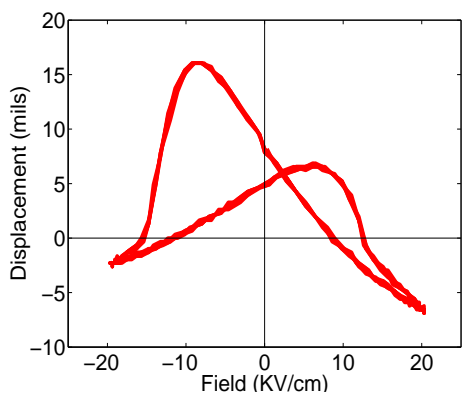

(b)

Figure 1: (a) THUNDER transducer, and (b) stress-dependent electromechanical behavior. 
A second technique for constructing macroscopic models is to employ thermodynamic or phenomenological principles directly at the macroscopic level to characterize measured phenomena. In the former category, states such as remanent polarization or remanent strain re-identified as internal variables and the first and second laws of thermodynamics are invoked to characterize material behavior $[8,33]$ - the reader is referred to Landis [10] for a summary of constitutive theories resulting from reversible and irreversible thermodynamics. Purely phenomenological approaches range from the rheology theory of [32] to Preisach models [6, 21]. We note that Preisach theory for ferroelectric materials is significantly less mature than its ferromagnetic counterpart and presently is focused on ferroelectric rather than ferroelastic switching mechanisms.

The present framework combines energy principles at the lattice level, theory of thermally activated processes, and stochastic homogenization techniques to characterize hysteresis due to ferroelectric and ferroelastic switching in a manner which facilitates material characterization, transducer design, and model-based control design. In the first step of the development, Helmholtz and Gibbs energy relations are constructed at the lattice level to quantify the internal and electrostatic energy associated with $90^{\circ}$ and $180^{\circ}$ dipole orientations. To characterize regimes in which thermal activation is significant, the Gibbs and relative thermal energies are balanced through Boltzmann theory to provide equilibrium relations quantifying local strains and polarizations as a function of input stresses and fields. As shown in $[24,31]$, these local relations reduce to minima of the Gibbs energy in the limit of negligible thermal activation - enforcement of these minimization criteria significantly improve the efficiency of the model and inversion process employed for linear control design in regimes where relaxation processes are negligible. This provides a model for homogeneous, single crystal compounds with uniform effective fields. To incorporate the effects of polycrystallinity, material nonhomogeneities, and variable effective fields, parameters such as the local coercive fields are considered to be manifestations of underlying distributions rather than constants. Homogenization in this manner yields low-order models posed in terms of effective parameters that are efficient to implement in optimization or control algorithms.

An important attribute of the framework is the flexibility it provides for characterizing hysteresis and constitutive nonlinearities in a broad range of ferroelectric, ferromagnetic and ferroelastic materials. This combination of energy analysis and theory of thermally activated processes had its genesis in the SMA models developed by Müller, Achenbach and Seelecke $[1,23]$. The incorporation of stochastic homogenization techniques and extension to ferroelectric [27, 31], ferromagnetic [25, 26], and polycrystalline SMA compounds $[14,15,22]$ has led to the formulation of a general framework for characterizing hysteresis in ferroic compounds $[24,29,30]$. The theory presented here extends this framework and the work presented in [9] by incorporating stress and field-induced $90^{\circ}$ switching as motivated by both fundamental material considerations and design criteria associated with stress-dependence as illustrated in Figure 1(b).

Physical mechanisms associated with ferroelectric and ferroelastic switching are summarized in Section 2 to motivate $90^{\circ}$ and $180^{\circ}$ switching mechanisms that need to be incorporated in energy relations. In Section 3, we summarize the construction of a Gibbs energy functional based on Landau-Devonshire principles. Minimization of this functional quantifies homogeneous, single crystal behavior in the absence of relaxation processes. Whereas efficient to formulate, the Landau-Devonshire functional is overly restrictive and often unstable to implement due to its reliance on high-order polynomials. To alleviate these restrictions, 2-D piecewise quadratic energy functionals, motivated by the LandauDevonshire formulation, are employed in Section 4 to provide local models characterizing ferroelectric and ferroelastic switching. In Section 5, stochastic homogenization techniques are employed to construct a macroscopic model for polycrystalline, nonhomogeneous materials and in Section 6, properties of the model are illustrated through comparison and prediction of experimental PLZT data. 


\section{Ferroelectric and Ferroelastic Switching Mechanisms}

To motivate mechanisms which must be incorporated in energy formulations, we summarize ferroelectric and ferroelastic switching mechanisms in ferroelectric compounds. We will focus primarily on $\mathrm{Pb}(\mathrm{Zr}, \mathrm{Ti}) \mathrm{O}_{3}$ (lead zirconate-titanate or $\mathrm{PZT}$ ), which is comprised of $\mathrm{PbZr}_{x-1} \mathrm{O}_{3}$ (lead zirconate) and $\mathrm{PbTi}_{x} \mathrm{O}_{3}$ (lead titanate) where $x$ is chosen to optimize electromechanical coupling, and lanthanum doped PZT (PLZT). For temperatures above the Curie point $T_{c}$, the structures of $\mathrm{PbTiO}_{3}$ and $\mathrm{PbZrO}_{3}$ are cubic whereas for $T<T_{c}$, the structure of $\mathrm{PbTiO}_{3}$ is tetragonal and $\mathrm{PbZrO}_{3}$ is orthorhombic [24]. We illustrate the switching mechanisms in the context of the paraelectric cubic and the ferroelectric tetragonal structure of lead titanate in Figure 2 and note the analogous behavior is observed for the orthorhombic structure of lead zirconate. To simplify the model while retaining the structure required for stress-induced switching, we consider a 2-D polarization $\mathbf{P}=\left(P_{1}, P_{3}\right)$ having the orientation shown in Figure 2(a).

Ferroelectric switching is induced by the application of an electric field $\mathbf{E}$ that is larger in magnitude than the coercive field $E_{c}$. For $\mathrm{PbTiO}_{3}$, this causes the central $\mathrm{Ti}^{+4}$ ion to relocate to a new equilibrium position, resulting in a $180^{\circ}$ change in polarization that is parallel to the applied field as depicted in Figure 3(a). Ferroelastic switching is caused by the application of a stress $\boldsymbol{\sigma}$ that is larger in magnitude than the coercive stress $\sigma_{c}$ producing a $90^{\circ}$ change in polarization that is perpendicular to the applied stress as illustrated in Figure 3(b). The ferroelectric and ferroelastic switching mechanisms cause a hysteretic relationship between input fields $\mathbf{E}$ and $\boldsymbol{\sigma}$ and output polarization $\mathbf{P}$ and strains $\boldsymbol{\varepsilon}$. Experimental data illustrating typical ferroelectric and ferroelastic response of the soft piezoelectric ceramic PLZT, can be found in [13].

Figures 4(a) and 4(b) illustrate the relationship between an externally applied field and the polarization and strains induced in a soft PLZT compound. At point A, the electric field is sufficiently strong so that all the dipoles form one domain that is aligned in the direction of the applied field. As the field is decreased, it approaches the negative coercive field in the region around point $\mathrm{B}$ where $180^{\circ}$ switching commences. Additionally, this often includes $90^{\circ}$ switching as indicated by the presence of a negative strain at point $\mathrm{B}$ as depicted in Figure 4(b). At point $\mathrm{C}$, all the dipoles have switched and again form one domain that is aligned in the direction of the electric field. At point $\mathrm{C}$, the polarization is opposite to that at point $\mathrm{A}$ whereas the strains have the same value. As the field is increased, $90^{\circ}$ switching occurs at point $\mathrm{D}$ and rapidly continues back to point $\mathrm{A}$ where the full $180^{\circ}$ switch has occurred and the dipoles are again aligned with the applied electric field.

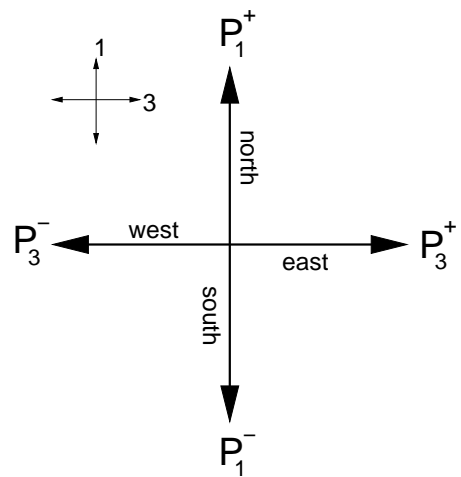

(a)

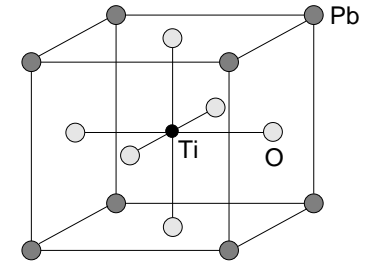

(b)

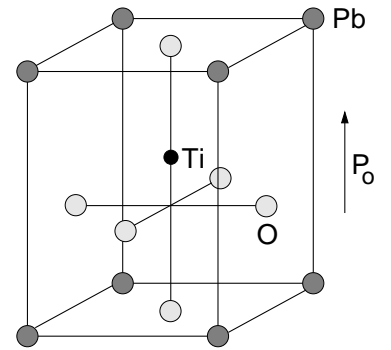

(c)

Figure 2: (a) Coordinate system, (b) high temperature paraelectric cubic form of $\mathrm{PbTiO}_{3}$, and (c) low temperature ferroelectric tetragonal form of lead titanate and resulting spontaneous polarization $P_{0}$. 


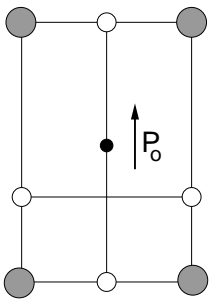

(a)

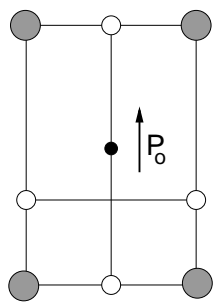

(b)

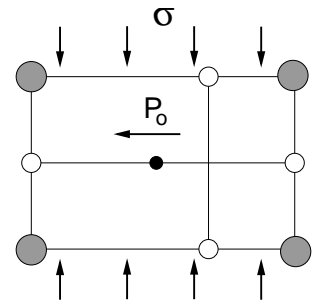

Figure 3: (a) Ferroelectric $180^{\circ}$ switch in spontaneous polarization $P_{0}$ induced by an applied electric field, and (b) ferroelastic $90^{\circ}$ switch induced by an applied stress.

Figures 4(c) and 4(d) illustrate the relationship between an externally applied stress and the polarization and strains induced in the soft PLZT. At point F, the dipoles are aligned in the positive 3 -direction and the material acts as one domain. As the compressive stress is increased in magnitude, it approaches the coercive stress. In the region around point $\mathrm{G}, 90^{\circ}$ switching occurs and the dipoles begin to align perpendicular to the direction of the applied stress. This is indicated by the presence of a negative strain at point $\mathrm{G}$ in Figure 4(d). As the stress is reduced in magnitude, the material remains poled perpendicular to the applied stress resulting in a decrease in the polarization in the 3-direction.

\section{Single Crystal Model: Landau-Devonshire Energy Relation}

To motivate issues which must be addressed when constructing energy relations and obtaining conditions necessary for equilibrium states, in the absence of thermal excitation, we first employ LandauDevonshire principles to construct a local model. This approach has the advantage of simple energy representations but is overly restrictive due to the requisite high-order polynomials. These restrictions are addressed in Section 4 where the Landau-Devonshire theory is used to motivate piecewise energy definitions. Using the theory of Landau and Devonshire, the Helmholtz energy is expressed as a truncated power series in term of the polarization with coefficients chosen to ensure measured material properties. As detailed in [24], the behavior of materials exhibiting first and second-order phase transitions can be quantified by the retention of $4^{\text {th }}$ and $6^{\text {th }}$ order polarization terms of the Helmholtz energy. Lead titanate and lead zirconate both exhibit first-order transitions near the transition temperature. However, we shall consider operating regimes $T<<T_{c}$, where the materials are actually employed for transduction; which necessitates retention of polarization terms only up to $4^{\text {th }}$ order.

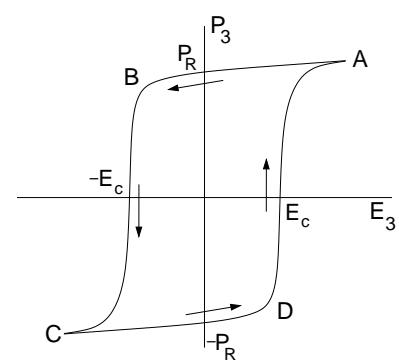

(a)

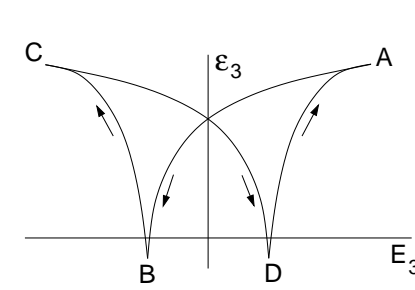

(b)

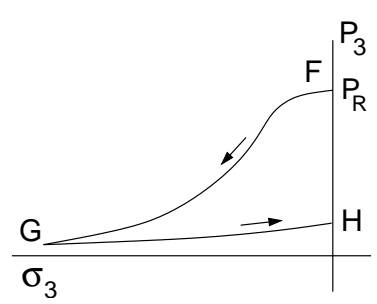

(c)

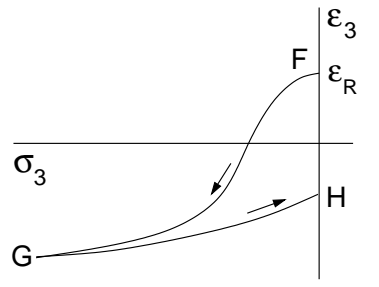

(d)

Figure 4: (a) Hysteretic field-polarization relation for bulk PLZT, (b) field-strain behavior of PLZT, (c) stress-polarization relation for PLZT, and (d) stress-strain behavior of PLZT. 
To model the internal free-energy of a single lead titanate crystal, we consider electric field $\mathbf{E}=$ $\left(E_{1}, E_{3}\right)$ and stress $\boldsymbol{\sigma}=\left(\sigma_{1}, \sigma_{3}\right)$ inputs and polarization $\mathbf{P}=\left(P_{1}, P_{3}\right)$ and strain $\boldsymbol{\varepsilon}=\left(\varepsilon_{1}, \varepsilon_{3}\right)$ outputs. The polarization component of the Helmholtz free-energy $\psi_{P}$ is taken to be the $4^{\text {th }}$ order polynomial

$$
\psi_{P}(\mathbf{P})=\alpha_{1} P_{1}^{2}+\alpha_{3} P_{3}^{2}+\alpha_{11} P_{1}^{4}+\alpha_{33} P_{3}^{4}+\alpha_{31} P_{3}^{2} P_{1}^{2} .
$$

The coefficients are chosen so that $\alpha_{i j}>0$ and $\alpha_{i}<0$ below the Curie point and can be related to physical properties of ferroelectric compounds such as the remanence polarization $P_{R}$ and coercive field $E_{c}-$ e.g., see (8) and (9). The electromechanical coupling component is given by

$$
\psi_{e s}(\mathbf{P}, \boldsymbol{\varepsilon})=-a_{1} \varepsilon_{1} P_{1}-a_{3} \varepsilon_{3} P_{3}-a_{13} \varepsilon_{1} P_{3}-a_{31} \varepsilon_{3} P_{1}-q_{1} \varepsilon_{1} P_{1}^{2}-q_{3} \varepsilon_{3} P_{3}^{2}-q_{13} \varepsilon_{1} P_{3}^{2}-q_{31} \varepsilon_{3} P_{1}^{2}
$$

where $a_{i j}$ are piezoelectric coupling coefficients and $q_{i j}$ are electrostrictive coupling coefficients. When shear effects are neglected, the elastic free-energy is

$$
\psi_{e l}(\varepsilon)=\frac{1}{2} Y_{1} \varepsilon_{1}^{2}+\frac{1}{2} Y_{3} \varepsilon_{3}^{2}
$$

where $Y_{i}$ are the components of the Young's modulus. The total Helmholtz free-energy is then given by

$$
\psi(\mathbf{P}, \boldsymbol{\varepsilon})=\psi_{P}(\mathbf{P})+\psi_{e s}(\mathbf{P}, \boldsymbol{\varepsilon})+\psi_{e l}(\boldsymbol{\varepsilon}) .
$$

The work due to an externally applied electric field and an applied stress is incorporated by employing a Gibbs energy of the form

$$
G(\mathbf{E}, \mathbf{P}, \boldsymbol{\sigma}, \boldsymbol{\varepsilon})=\psi(\mathbf{P}, \boldsymbol{\varepsilon})-\mathbf{E} \cdot \mathbf{P}-\boldsymbol{\sigma} \cdot \boldsymbol{\varepsilon}
$$

As detailed in $[3,24]$, necessary conditions for minimizing the Gibbs energy in the absence of thermal relaxation mechanisms are

$$
\frac{\partial G}{\partial \varepsilon_{1}}=0, \quad \frac{\partial G}{\partial \varepsilon_{3}}=0, \quad \frac{\partial G}{\partial P_{1}}=0, \quad \frac{\partial G}{\partial P_{3}}=0 .
$$

These conditions specify how the polarization and strains change to minimize the internal energy when an external force is applied and thermal activation is negligible. The condition $\frac{\partial G}{\partial \varepsilon_{1}}=0$ implies that

$$
\varepsilon_{1}=Y_{1}^{-1}\left(\sigma_{1}+a_{1} P_{1}+a_{13} P_{3}+q_{1} P_{1}^{2}+q_{13} P_{3}^{2}\right)
$$

and $\frac{\partial G}{\partial \varepsilon_{3}}=0$ implies that

$$
\varepsilon_{3}=Y_{3}^{-1}\left(\sigma_{3}+a_{3} P_{3}+a_{31} P_{1}+q_{3} P_{3}^{2}+q_{31} P_{1}^{2}\right) .
$$

These relations allow us to determine explicit expressions for the strains. In a manner analogous to that employed in [19] for a stress-free state, we substitute equations (4) and (5) into the Gibbs energy (3) to directly couple the stress and the polarization. This yields

$$
G(\mathbf{E}, \mathbf{P}, \boldsymbol{\sigma})=\widehat{\psi}(\mathbf{P}, \boldsymbol{\sigma})-\mathbf{E} \cdot \mathbf{P}
$$

where $\widehat{\psi}(\mathbf{P}, \boldsymbol{\sigma})$ is the fourth order polynomial

$$
\begin{aligned}
\widehat{\psi}(\mathbf{P}, \sigma)= & \gamma_{1} P_{1}^{4}+\gamma_{2} P_{3}^{4}+\gamma_{3} P_{3}^{2} P_{1}^{2}+\gamma_{4} P_{1}^{3}+\gamma_{5} P_{3}^{3}+\gamma_{6} P_{1}^{2} P_{3}+\gamma_{7} P_{1} P_{3}^{2} \\
& +\gamma_{8} P_{1}^{2}+\gamma_{9} P_{3}^{2}+\gamma_{10} P_{3} P_{1}+\gamma_{11} P_{1}+\gamma_{12} P_{3}+\gamma_{13} .
\end{aligned}
$$




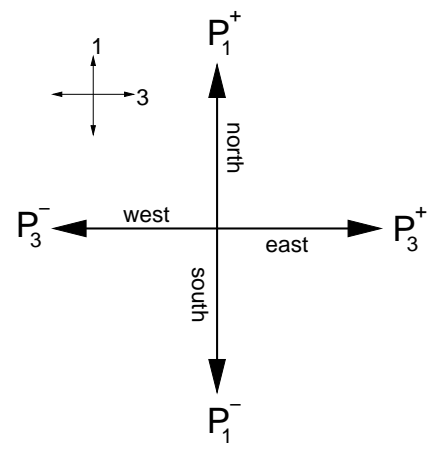

(a)

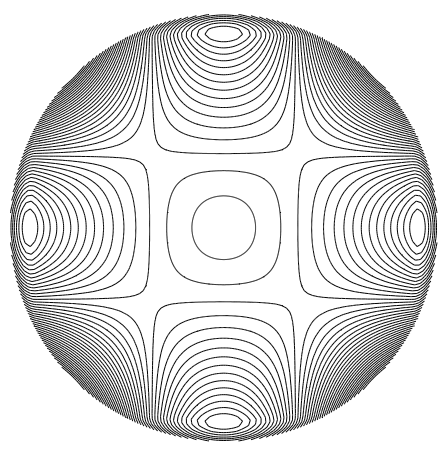

(b)

Figure 5: Gibbs energy landscape. (a) Coordinate system of energy landscape, and (b) contour plot of Gibbs energy landscape.

The Gibbs energy at zero stress and zero electric field has four local minima each corresponding to four energetically favorable polarization states $\pm P_{1}$ and $\pm P_{3}$ and is illustrated in Figure 5. Given an input pair $(\mathbf{E}, \boldsymbol{\sigma})$, the local polarization in the absence of thermal excitation can then be calculated by locally minimizing the Gibbs energy to obtain

$$
\langle\mathbf{P}\rangle=\arg \min _{\mathbf{P}} G(\mathbf{E}, \mathbf{P}, \boldsymbol{\sigma}) .
$$

The ferroelastic model thus quantifies the $\left(P_{1}, P_{3}\right)$ pair that minimizes the total energy of the system and the corresponding strain pair $\left(\varepsilon_{1}, \varepsilon_{3}\right)$ is designated by equations (4) and (5).

As noted previously, several of the coefficients in the ferroelastic model are related to physical material properties, thus allowing a means of determining their values. The remanent polarization $P_{R}$ and coercive field $E_{c}$ are computed by considering a stress-free Gibbs energy

$$
G(\mathbf{E}, \mathbf{P})=\psi(\mathbf{P})-\mathbf{E} \cdot \mathbf{P}
$$

and noting that the conditions $P_{1}=0$ and $\frac{\partial G}{\partial P_{3}}=0$ imply

$$
4 \alpha_{33} P_{3}^{3}+2 \alpha_{3} P_{3}-E_{3}=0 .
$$

As outlined in [34], equation (7) has three real roots, of which at least two are equal if the discriminant is 0 . The coercive field value for which this property is satisfied is

$$
E_{c}=\sqrt{\frac{-4^{3} \alpha_{3}^{3}}{6^{3} \alpha_{33}}} .
$$

When $E_{3}=0$, the remanent polarization determined from solving ( 7$)$ is given by

$$
P_{R}=\sqrt{\frac{-\alpha_{3}}{2 \alpha_{33}}} .
$$

Equations (8) and (9) provide a system of two equations and two unknowns sufficient to solve for $\alpha_{3}$ and $\alpha_{33}$ given values for $E_{c}$ and $P_{R}$.

A similar process may be applied to find $\alpha_{1}$ and $\alpha_{11}$ if the energy landscape is known to be nonsymmetric. Whereas some of the coefficents of this Landau-Devonshire formulation are related to physical quantities, it is difficult to relate all parameters with material properties. Furthermore, the model proves overly restrictive to implement in various regimes due to the high order polymonials. In the next sections, we present two piecewise-defined ferroelastic switching models that accurately describe the physics and yield methods for predicting material parameters. 


\section{Single Crystal Model: Piecewise Quadratic Energy Relations}

The high-order polynomials in the Landau-Devonshire theory of Section 3 can induce unstable behavior through small modifications of the coefficients. To facilitate model implementation, improve computational efficiency and increase the flexibility, we employ piecewise quadratic energy functionals which are approximations of the energy functionals based on Landau-Devonshire principles. The model coefficients directly relate to measured material properties allowing a means for parameter identification and estimation. The low-order piecewise polynomial models also facilitate transducer and control design.

\subsection{2-D Ferroelectric Switching Model}

We summarize first a 2-D ferroelectric switching model which characterizes the hysteretic fieldpolarization and field-strain relations depicted in Figures $4(\mathrm{a})$ and $4(\mathrm{~b})$ and allows $90^{\circ}$ polarization switching to occur when energetically favorable. However, the ferroelectric model does not characterize the stress-induced switching mechanisms resulting in the stress-polarization and stress-strain relations depicted in Figures 4(c) and 4(d) and should be limited to low stress regimes. The extension of the model to incorporate ferroelastic switching is addressed in Section 4.2.

\subsubsection{Helmholtz and Gibbs Energy Relations}

For fixed temperatures, we expand on the theory of [31] where it is illustrated that a reasonable expression for the Helmholtz energy is a piecewise quadratic relation. In the context of the model presented in Section 3, this is obtained by retaining $2^{\text {nd }}$ order terms of the Taylor approximations for the $4^{\text {th }}$ order polynomial expressions about the equilibria. To account for the $180^{\circ}$ and $90^{\circ}$ polarization switching, we allow four possible polarization orientations $\left(P_{1}^{ \pm}, P_{3}^{ \pm}\right)$as depicted in Figure 5 . We define piecewise quadratic polynomials along the $P_{1}$ and $P_{3}$ directions to obtain the functionals

$$
\begin{aligned}
& \psi_{1}\left(P_{1}\right)=\left\{\begin{array}{lll}
\frac{1}{2} \eta_{1}\left(P_{1}+P_{1 R}\right)^{2} & , \quad P_{1} \leq-P_{1 I} \\
\frac{1}{2} \eta_{1}\left(P_{1}-P_{1 R}\right)^{2} & , \quad P_{1} \geq P_{1 I} \\
\frac{1}{2} \eta_{1}\left(P_{1 I}-P_{1 R}\right)\left(\frac{P_{1}^{2}}{P_{1 I}}-P_{1 R}\right) & , & \left|P_{1}\right|<P_{1 I}
\end{array}\right. \\
& \psi_{3}\left(P_{3}\right)=\left\{\begin{array}{lll}
\frac{1}{2} \eta_{3}\left(P_{3}+P_{3 R}\right)^{2} & , \quad P_{3} \leq-P_{3 I} \\
\frac{1}{2} \eta_{3}\left(P_{3}-P_{3 R}\right)^{2} & , \quad P_{3} \geq P_{3 I} \\
\frac{1}{2} \eta_{3}\left(P_{3 I}-P_{3 R}\right)\left(\frac{P_{3}^{2}}{P_{3 I}}-P_{3 R}\right) & , & \left|P_{3}\right|<P_{3 I}
\end{array}\right.
\end{aligned}
$$

where $P_{1 I}$ and $P_{3 I}$ denote the positive inflection points in the $P_{1}$ and $P_{3}$ directions and $P_{1 R}$ and $P_{3 R}$ denote the polarization at which the positive minimum occurs in the $P_{1}$ and $P_{3}$ directions. The 2-D Helmholtz energy is then defined by

$$
\psi_{p}(\mathbf{P})=\psi_{1}\left(P_{1}\right)+\psi_{3}\left(P_{3}\right)+c P_{1}^{2} P_{3}^{2} .
$$

The resulting Gibbs free energy, as illustrated in Figure 5, quantifying the change in the energy landscape due to an applied field, is provided by the inclusion of the electrostatic work term and results in

$$
G(\mathbf{E}, \mathbf{P})=\psi_{p}(\mathbf{P})-\mathbf{E} \cdot \mathbf{P}
$$




\subsubsection{Switching in the Absence of Thermal Activation}

In the absence of thermal activation, ferroelectric dipole switching occurs when the magnitude of the applied electric field is greater than that of the coercive field $E_{c}$. From an energy perspective, the polarization switching occurs when the applied electric field is sufficiently large to eliminate local minima in the Gibbs energy. The local polarization $\langle\mathbf{P}\rangle$ of the single crystal can be calculated by solving the necessary conditions

$$
\frac{\partial G}{\partial P_{1}}=0, \quad \frac{\partial G}{\partial P_{3}}=0
$$

Alternatively, given an input field $\mathbf{E}$, the local polarization can be found by locally minimizing the Gibbs energy,

$$
\langle\mathbf{P}\rangle=\arg \min _{\mathbf{P}} G(\mathbf{E}, \mathbf{P})
$$

where $G$ is defined by equation (11). Since the single crystal has a dipole structure that will change orientation to minimize the internal energy, the order in which the minima are eliminated determines the type of ferroelectric switching that occurs.

\section{Ferroelectric $90^{\circ}$ Switching}

A ferroelectric $90^{\circ}$ switch is illustrated in Figure 6(a) where an applied field has caused the $P_{3}^{-}$minima to vanish while the $P_{1}^{ \pm}$minima remain. If the single crystal was oriented in the $P_{3}^{-}$direction, this would result in a $90^{\circ}$ switch to one of the neighboring wells in the $P_{1}$ direction. Given an applied electric field $E_{3}$, we can calculate the field necessary to induce switching by first solving the necessary condition $\frac{\partial G}{\partial P_{3}}=0$. We are calculating the value of the electric field that will make the $P_{3}^{-}$minima disappear, or equivalently the electric field that will make $P_{3}=P_{3 I}$. This occurs when $E_{c}=\eta_{3}\left(P_{3 R}-P_{3 I}\right)+c P_{1}^{2} P_{3 I}$. However, the second necessary condition $\frac{\partial G}{\partial P_{1}}=0$ requires that $P_{1}=0$ since we assume the applied field has no $E_{1}$ component. This results in

$$
E_{c}=\eta_{3}\left(P_{3 R}-P_{3 I}\right) .
$$

The electric field required to eliminate the minima in the $90^{\circ}$ orientation is denoted by $E_{c}^{90}$. For a ferroelectric $90^{\circ}$ switch to occur, $E_{c}^{90}>E_{c}$.

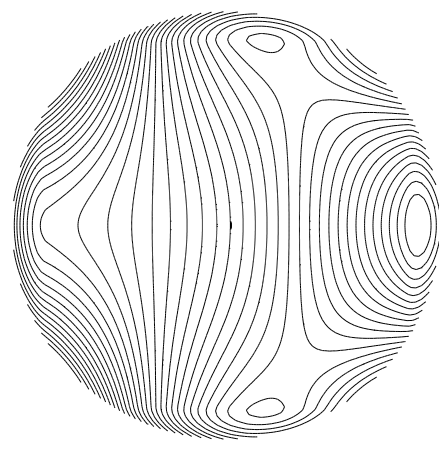

(a)



Figure 6: (a) Elimination of $180^{\circ}$ minimum through application of an applied field greater than $E_{c}$, and (b) $90^{\circ}$ minima can disappear before the $180^{\circ}$ minima disappears if $E_{c}^{90}<E_{c}$. 


\section{Ferroelectric $180^{\circ}$ Switching}

As detailed in Section $2,180^{\circ}$ polarization switching due to an applied electric field can result from a direct $180^{\circ}$ switch (e.g., $P_{3}^{-} \rightarrow P_{3}^{+}$) or a pair of $90^{\circ}$ switches (e.g., $P_{3}^{-} \rightarrow P_{1}^{ \pm} \rightarrow P_{3}^{+}$). The $180^{\circ}$ switching mechanism which consists of a pair of $90^{\circ}$ switches occurs when $\left|E_{3}\right| \geq E_{c}^{90}>E_{c}$. The direct $180^{\circ}$ switch is illustrated in Figure 6(b) where $E_{c}>E_{c}^{90}$ and the applied field has caused the $P_{3}^{-}$and $P_{1}^{ \pm}$minima to vanish. If the single crystal was oriented in the $P_{3}^{-}$direction, this would result in a direct $180^{\circ}$ switch to the well in the $P_{3}^{+}$direction.

\subsubsection{Switching in the Presence of Thermal Activation}

In the model developed in Section 4.1.2, dipole switching occurred only when the magnitude of the applied electric field exceeded that of the coercive field value of the single crystal. However, mechanisms such as excitation from thermal effects can induce switching before local minima are eliminated. To model this phenomena, we incorporate the effects of thermal activation. We continue to assume that the material is homogeneous and that each dipole in this collection has the same energy landscape and are subject to the same switching mechanisms. This allows us to model the switching that occurs by evolving the fraction of dipoles in each allowed orientation by a population model that is similar to what is derived in [31].

As detailed in [24], the Gibbs energy and the relative thermal energy $\frac{k T}{V}$ is balanced through the Boltzmann relation

$$
\mu(G(\mathbf{P}))=C e^{-G(\mathbf{P}) V / k T} .
$$

Here $V$ denotes a representative control volume, $k$ is Boltzmann's constant and $T$ is the temperature. The fraction of dipoles in each allowed dipole orientation is given by $x_{3}^{+}, x_{3}^{-}, x_{1}^{+}$, and $x_{1}^{-}$. The average polarization associated with each allowed dipole orientation (the minima of the Gibbs energy) is denoted by $\left\langle\mathbf{P}_{\mathbf{3}}^{+}\right\rangle,\left\langle\mathbf{P}_{\mathbf{3}}^{-}\right\rangle,\left\langle\mathbf{P}_{\mathbf{1}}^{+}\right\rangle$, and $\left\langle\mathbf{P}_{\mathbf{1}}^{-}\right\rangle$. It should be noted that these are vector quantities and have polarization values in both the $P_{1}$ and $P_{3}$ directions. Conservation of the total number of dipoles yields

$$
x_{3}^{+}+x_{3}^{-}+x_{1}^{+}+x_{1}^{-}=1 .
$$

For a homogeneous material, the components of the local polarization

$$
\langle\mathbf{P}\rangle=\left(P_{1}, P_{3}\right)
$$

are given by

$$
\begin{aligned}
& P_{3}=x_{3}^{+}\left\langle P_{3}^{+}\right\rangle_{3}+x_{3}^{-}\left\langle P_{3}^{-}\right\rangle_{3}+x_{1}^{+}\left\langle P_{1}^{+}\right\rangle_{3}+x_{1}^{-}\left\langle P_{1}^{-}\right\rangle_{3} \\
& P_{1}=x_{3}^{+}\left\langle P_{3}^{+}\right\rangle_{1}+x_{3}^{-}\left\langle P_{3}^{-}\right\rangle_{1}+x_{1}^{+}\left\langle P_{1}^{+}\right\rangle_{1}+x_{1}^{-}\left\langle P_{1}^{-}\right\rangle_{1}
\end{aligned}
$$

where the vector components of the minima are denoted by the subscripts 1 or 3 .

The likelihood of switching from the $P_{1}^{-}$orientation (south) to the $P_{3}^{+}$(east) is specified by $p_{s e}$ and is calculated by

$$
p_{s e}=\frac{\gamma}{\tau} \frac{1}{e^{-\gamma\left(G\left(\mathbf{P}_{\text {min }}^{s}\right)-G\left(\mathbf{P}_{\text {barrier }}^{s e}\right)\right)}-1}
$$

where the relaxation time $\tau$ is the reciprocal of the frequency at which dipoles attempt a switch and $\gamma=\frac{V}{k T}$. Here, $\mathbf{P}_{\text {barrier }}^{\text {se }}$ denotes the location of the saddle point from the $P_{1}^{-}$orientation (south) to $P_{3}^{+}$(east) and $G\left(\mathbf{P}_{\text {barrier }}^{\text {se }}\right)$ is the Gibbs energy evaluated at that point. Similarly, $\mathbf{P}_{\text {min }}^{s}$ denotes the location of the south minima and $G\left(\mathbf{P}_{\min }^{s}\right)$ is the Gibbs energy evaluated at that point. 
The likelihood of switching from the $P_{1}^{+}$orientation (north) to the $P_{3}^{+}$(east) is specified by $p_{n e}$ and is calculated by

$$
p_{n e}=\frac{\gamma}{\tau} \frac{1}{e^{-\gamma\left(G\left(\mathbf{P}_{\text {min }}^{n}\right)-G\left(\mathbf{P}_{\text {barrier }}^{n e}\right)\right)}-1}
$$

where $\mathbf{P}_{\text {barrier }}^{\text {ne }}$ denotes the location of the saddle point from the $P_{1}^{+}$orientation (north) to $P_{3}^{+}$(east) and $G\left(\mathbf{P}_{\text {barrier }}^{n e}\right)$ is the Gibbs energy evaluated at that point. Similarly, $\mathbf{P}_{\text {min }}^{n}$ denotes the location of the north minima and $G\left(\mathbf{P}_{\min }^{n}\right)$ is the Gibbs energy evaluated at that point. The remaining likelihoods follow similarly and have the notation summarized in Table 1.

Finally, the evolution of the dipole fractions due to thermal activation is governed by the system of differential equations

$$
\begin{aligned}
& \dot{x}_{3}^{+}=p_{s e} x_{1}^{-}+p_{n e} x_{1}^{+}-p_{e s} x_{3}^{+}-p_{e n} x_{3}^{+} \\
& \dot{x}_{3}^{-}=p_{s w} x_{1}^{-}+p_{n w} x_{1}^{+}-p_{w s} x_{3}^{-}-p_{w n} x_{3}^{-} \\
& \dot{x}_{1}^{+}=p_{w n} x_{3}^{-}+p_{e n} x_{3}^{+}-p_{n w} x_{1}^{+}-p_{n e} x_{1}^{+} \\
& \dot{x}_{1}^{-}=p_{w s} x_{3}^{-}+p_{e s} x_{3}^{+}-p_{s w} x_{1}^{-}-p_{s e} x_{1}^{-} .
\end{aligned}
$$

The dimension of this system of first order ordinary differential equations can be reduced by using (15) and solved quickly and with sufficient accuracy using an implicit method such as backward Euler method. To compute the transition likelihoods, values of the minima and the saddle points of the energy landscape are computed using Newton's method with line search to solve for the location where the gradient of the Gibbs energy is zero.

\subsection{2-D Ferroelastic Switching Model}

Here we extend the framework of Section 4.1 to construct a ferroelastic switching model which quantifies the hysteretic field-polarization and field-strain relationships depicted in Figures 4(a) and 4(b) and also characterizes the hysteretic stress-polarization and stress-strain relationships depicted in Figures 4(c) and 4(d). The ferroelastic model extends the phenomenological Landau-Devonshire energy model developed in [2] and summarized in Section 3 through the construction of piecewise quadratic energy functionals.

\subsubsection{Helmholtz and Gibbs Energy Relations}

To model stress-induced switching in ferroelectric materials, we again consider electric field $\mathbf{E}=$ $\left(E_{1}, E_{3}\right)$ and stress $\boldsymbol{\sigma}=\left(\sigma_{1}, \sigma_{3}\right)$ inputs and polarization $\mathbf{P}=\left(P_{1}, P_{3}\right)$ and strain $\boldsymbol{\varepsilon}=\left(\varepsilon_{1}, \varepsilon_{3}\right)$ outputs.

\begin{tabular}{c|c} 
State Transition & notation \\
\hline$P_{1}^{-} \rightarrow P_{3}^{+}$ & $p_{s e}$ \\
$P_{1}^{-} \rightarrow P_{3}^{-}$ & $p_{s w}$ \\
$P_{1}^{+} \rightarrow P_{3}^{+}$ & $p_{n e}$ \\
$P_{1}^{+} \rightarrow P_{3}^{-}$ & $p_{n w}$ \\
$P_{3}^{-} \rightarrow P_{1}^{+}$ & $p_{w n}$ \\
$P_{3}^{-} \rightarrow P_{1}^{-}$ & $p_{w s}$ \\
$P_{3}^{+} \rightarrow P_{1}^{+}$ & $p_{e n}$ \\
$P_{3}^{+} \rightarrow P_{1}^{-}$ & $p_{w s}$ \\
\hline
\end{tabular}

Table 1: 2-D transition likelihoods. 
The polarization component of the Helmholtz free-energy $\psi_{P}$ is defined piecewise by (10) and is a function of the four possible polarization orientations $\left(P_{1}^{ \pm}, P_{3}^{ \pm}\right)$. The electromechanical coupling component $\psi_{e s}$ is given by (1). Ignoring shear effects, the elastic free-energy $\psi_{e l}$ is given by (2). The total Helmholtz free-energy is then

$$
\psi(\mathbf{P}, \boldsymbol{\varepsilon})=\psi_{p}(\mathbf{P})+\psi_{e s}(\mathbf{P}, \boldsymbol{\varepsilon})+\psi_{e l}(\boldsymbol{\varepsilon})
$$

and the Gibbs energy is

$$
G(\mathbf{E}, \mathbf{P}, \boldsymbol{\sigma}, \boldsymbol{\varepsilon})=\psi(\mathbf{P}, \boldsymbol{\varepsilon})-\mathbf{E} \cdot \mathbf{P}-\boldsymbol{\sigma} \cdot \boldsymbol{\varepsilon} .
$$

As detailed in Section 3 in the context of the Landau-Devonshire functional, the necessary conditions allow us to determine explicit expressions for the strains. This is a direct result of neglecting the shear terms in $\psi_{e l}$ which allows the strains to decouple. Substituting equations for the strains into the Gibbs energy (17) yields

$$
G(\mathbf{E}, \mathbf{P}, \boldsymbol{\sigma})=\widehat{\psi}(\mathbf{P}, \boldsymbol{\sigma})-\mathbf{E} \cdot \mathbf{P}
$$

where $\widehat{\psi}(\mathbf{P}, \boldsymbol{\sigma})$ is a fourth-order piecewise polynomial defined by

$$
\begin{aligned}
\widehat{\psi}(\mathbf{P}, \boldsymbol{\sigma})= & \gamma_{1} P_{1}^{4}+\gamma_{2} P_{3}^{4}+\gamma_{3} P_{3}^{2} P_{1}^{2}+\gamma_{4} P_{1}^{3}+\gamma_{5} P_{3}^{3}+\gamma_{6} P_{1}^{2} P_{3}+\gamma_{7} P_{1} P_{3}^{2} \\
& +\gamma_{8} P_{1}^{2}+\gamma_{9} P_{3}^{2}+\gamma_{10} P_{3} P_{1}+\gamma_{11} P_{1}+\gamma_{12} P_{3}+\gamma_{13},
\end{aligned}
$$

with the $\gamma_{i}$ coefficients defined in Appendix A.

\subsubsection{Local Polarization Model}

In the absence of thermal effects, the local polarization $\langle\mathbf{P}\rangle$ with an applied stress can be determined by solving the necessary conditions

$$
\frac{\partial G}{\partial P_{1}}=0, \quad \frac{\partial G}{\partial P_{3}}=0
$$

or by minimizing the Gibbs energy

$$
\langle\mathbf{P}\rangle=\arg \min _{\mathbf{P}} G(\mathbf{E}, \mathbf{P}, \boldsymbol{\sigma})
$$

where the Gibbs energy is defined by equation (18). To incorporate thermal relaxation, the local average polarization can be calculated by using the thermal evolution model developed in Section 4.1.3.

To calculate the value of the coercive field $E_{c}$ for the stress-dependent ferroelastic model, we apply

the necessary condition $\frac{\partial G}{\partial P_{3}}=0$ and solve for the value of the electric field that eliminates the minima in the Gibbs energy. This yields

$$
\begin{aligned}
E_{c}\left(\sigma_{3}\right)= & \frac{2 q_{3} P_{3 I}-a_{3}}{Y_{3}}\left(\sigma_{3}\right)+\frac{2 Y_{3} q_{13}^{2}+2 Y_{1} q_{3}^{2}}{Y_{3} Y_{1}} P_{3 I}^{3}-\frac{3 Y_{3} a_{13} q_{13}+3 Y_{1} a_{3} q_{3}}{Y_{3} Y_{1}} P_{3 I}^{2}+ \\
& \frac{Y_{1} a_{3}^{2}+Y_{3} a_{13}^{2}}{Y_{3} Y_{1}} P_{3 I}+\eta_{3}\left(P_{3 R}-P_{3 I}\right) .
\end{aligned}
$$

It is clear from equation (20) that the coercive field is stress-dependent. A ferroelastic switch will occur when the applied stress is greater in magnitude than the coercive stress $\sigma_{c}$. To calculate $\sigma_{c}$, we set $E_{c}=0$ in equation (20) resulting in

$$
\begin{aligned}
\sigma_{c}= & \frac{Y_{3}}{a_{3}-2 q_{3} P_{3 I}}\left[\frac{2 Y_{3} q_{13}^{2}+2 Y_{1} q_{3}^{2}}{Y_{3} Y_{1}} P_{3 I}^{3}-\frac{3 Y_{3} a_{13} q_{13}+3 Y_{1} a_{3} q_{3}}{Y_{3} Y_{1}} P_{3 I}^{2}+\right. \\
& \left.\frac{Y_{1} a_{3}^{2}+Y_{3} a_{13}^{2}}{Y_{3} Y_{1}} P_{3 I}+\eta_{3}\left(P_{3 R}-P_{3 I}\right)\right]
\end{aligned}
$$




\section{$4.3 \quad$ 1-D Ferroelastic Switching Model}

To provide a simplified model which facilitates real-time implementation for transducer and control design, we present here a 1-D model that incorporates both $90^{\circ}$ ferroelectric and ferroelastic polarization switching mechanisms. For the 1-D input fields considered in the examples of Section 6 , the 1-D ferroelastic model has a $90^{\circ}$ switching behavior similar to the 2-D ferroelastic model and is more computationally efficient. We note that aspects of the functionals are similar to those employed for SMA undergoing austinite - martinsite phase transformations and the reader is referred to [14, 24, 29] for details illustrating properties of the SMA relations.

\subsubsection{Helmholtz and Gibbs Energy Relations}

We consider electric field and stress inputs $(E, \sigma)$ and polarization and strain outputs $(P, \varepsilon)$. The polarization has three allowed dipole states $P_{-}, P_{+}$and $P_{90}$ as shown in Figure 7 . The values $P_{-}$and $P_{+}$correspond to the $\pm P_{3}$ orientation in the 2-D model while $P_{90}$ represents both $\pm P_{1}$. We define the polarization component of the Helmholtz free energy to be,

$$
\psi_{p}(P)= \begin{cases}\frac{\eta}{2}\left(P+P_{R}\right)^{2} & , \quad P \leq-P_{I} \\ \frac{\eta_{1}}{2}\left(P+P_{m}\right)^{2}+\beta & , \quad-P_{I}<P<-P_{90 I} \\ \frac{\eta_{2}}{2}(P)^{2}+\Delta & , \quad|P| \leq P_{90 I} \\ \frac{\eta_{1}}{2}\left(P-P_{m}\right)^{2}+\beta & , \quad P_{90 I}<P<P_{I} \\ \frac{\eta}{2}\left(P-P_{R}\right)^{2} & , \quad P \geq P_{I}\end{cases}
$$

where

$$
\begin{array}{ll}
P_{m}=\frac{\eta\left(P_{I}-P_{R}\right) P_{90 I}-\eta_{2} P_{90 I} P_{I}}{\eta\left(P_{I}-P_{R}\right)-\eta_{2} P_{90 I}}, & \eta_{1}=\eta \frac{P_{I}-P_{R}}{P_{I}-P_{m}} \\
\beta=\frac{\eta}{2}\left(P_{I}-P_{R}\right)^{2}-\frac{\eta_{1}}{2}\left(P_{I}-P_{m}\right)^{2}, & \Delta=\frac{\eta_{1}}{2}\left(P_{90 I}-P_{m}\right)^{2}+\beta-\frac{\eta_{2}}{2}\left(P_{90 I}\right)^{2} .
\end{array}
$$

The electromechanical coupling component is given by

$$
\psi_{e s}(P, \varepsilon)=-a \varepsilon P-q \varepsilon P^{2}
$$

where $a$ is the piezoelectric coupling coefficient and $q$ is the electrostrictive coupling coefficient. The elastic free-energy is

$$
\psi_{e l}(\varepsilon)=\frac{1}{2} Y \varepsilon^{2}
$$



Figure 7: 1-D Gibbs energy with three minima corresponding to the three allowed dipole states $P_{-}, P_{+}$ and $P_{90}$. The 1-D energy has four inflection points $\pm P_{I}, \pm P_{90 I}$ and two local maxima (1-D saddle points) $\pm P_{s}$. 
where $Y$ is the Young's modulus. The total Helmholtz free-energy is then given by

$$
\psi(P, \varepsilon)=\psi_{p}(P)+\psi_{e s}(P, \varepsilon)+\psi_{e l}(\varepsilon) .
$$

Balancing the internal energy $\psi$ and the externally applied energy yields the Gibbs free energy

$$
G(E, P, \sigma, \varepsilon)=\psi(P, \varepsilon)-E P-\sigma \varepsilon .
$$

The necessary condition $\frac{\partial G}{\partial \varepsilon}=0$ yields

$$
\varepsilon=Y^{-1}\left(\sigma+a P+q P^{2}\right) .
$$

Following the same procedure as detailed in Section 3, the strain is substituted into the Gibbs energy (23) resulting in

$$
G(E, P, \sigma)=\widehat{\psi}(P, \sigma)-E P-\sigma \varepsilon
$$

\subsubsection{Switching in the Absence of Thermal Activation}

Neglecting thermal effects, the local polarization $\langle P\rangle$ for a single crystal with an applied stress can be determined by solving the necessary conditions

$$
\frac{\partial G}{\partial P}=0
$$

or by minimizing the Gibbs energy

$$
\langle P\rangle=\arg \min _{P} G(E, P, \sigma)
$$

where the Gibbs energy is defined by equation (24).

To calculate the value of the coercive field, we apply the necessary condition $\frac{\partial G}{\partial P}=0$ and solve for the value of the electric field that results in $P=P_{I}$. This yields

$$
E_{c}(\sigma)=\frac{2 q P_{I}-a}{Y}(\sigma)+\frac{2 q^{2} P_{I}^{3}}{Y}-\frac{3 a q P_{I}^{2}}{Y}+\frac{a^{2} P_{I}}{Y}+\eta\left(P_{R}-P_{I}\right) .
$$

It is clear from (26) that the coercive field is stress dependent. The coercive stress $\sigma_{c}$ can be computed by setting $E_{c}=0$ in (26). This results in

$$
\sigma_{c}=\frac{1}{a-2 q P_{I}}\left[\eta Y\left(P_{R}-P_{I}\right)+2 q^{2}\left(P_{I}\right)^{3}-3 a q\left(P_{I}\right)^{2}+a^{2}\left(P_{I}\right)\right] .
$$

The electric field required to eliminate the $90^{\circ}$ minima in the Gibbs energy is denoted by $E_{c}^{90}$. An explicit expression for the value of $E_{c}^{90}$ is found to be

$$
E_{c}^{90}(\sigma)=-(\sigma) \frac{a+2 q P_{90}}{Y}-\frac{2 q^{2} P_{90}^{3}+3 a q P_{90}^{2}+\left(a^{2}-\eta_{2} Y\right) P_{90}}{Y} .
$$

As in the $2-\mathrm{D}$ model, a direct $180^{\circ}$ ferroelectric polarization switch (i.e., $P_{ \pm} \rightarrow P_{\mp}$ ) can occur if $E_{c}^{90}<E_{c}$. A $90^{\circ}$ ferroelectric polarization switch (i.e., $P_{ \pm} \rightarrow P_{90}$ ) can occur if $E_{c}^{90}>E_{c}$ and a $90^{\circ}$ ferroelastic polarization switch will occur when $\sigma>\sigma_{c}$. 


\subsubsection{Switching in the Presence of Thermal Activation}

In the manner detailed in Section 4.1.3, thermal activation processes are incorporated through the use of the Boltzmann relation (14). For the 1-D model, the polarization has three allowed dipole states $P_{-}, P_{+}$and $P_{90}$. The fraction of dipoles in each allowed orientation is given by $x_{-}, x_{90}$ and $x_{+}$. Conservation of the number of dipoles yields

$$
x_{-}+x_{90}+x_{+}=1 .
$$

The local polarization $\langle P\rangle$ is given by

$$
\langle P\rangle=x_{-}\left\langle P_{-}\right\rangle+x_{90}\left\langle P_{90}\right\rangle+x_{+}\left\langle P_{+}\right\rangle
$$

where $\left\langle P_{-}\right\rangle,\left\langle P_{90}\right\rangle$ and $\left\langle P_{+}\right\rangle$are the expected polarization values associated with each allowed dipole orientation. As illustrated in $[24,31]$, these values are found by integrating the product of the polarization $P$ and the Boltzmann probability density $\mu(G(P))$ over the allowed polarization states. This simplifies to the relations

$$
\left\langle P_{-}\right\rangle=\frac{\int_{-\infty}^{-P_{I}} P e^{-\gamma G} d P}{\int_{-\infty}^{-P_{I}} e^{-\gamma G} d P}, \quad\left\langle P_{90}\right\rangle=\frac{\int_{-P_{90 I}}^{P_{90 I}} P e^{-\gamma G} d P}{\int_{-P_{90 I}}^{P_{90 I}} e^{-\gamma G} d P},\left\langle P_{+}\right\rangle=\frac{\int_{P_{I}}^{\infty} P e^{-\gamma G} d P}{\int_{P_{I}}^{\infty} e^{-\gamma G} d P}
$$

where $\gamma=\frac{V}{k T}$. For computational efficiency, we can approximate these values by use of the necessary condition $\frac{\partial G}{\partial P}=0$ which yields

$$
\left\langle P_{-}\right\rangle=\frac{E}{\eta}-P_{R}, \quad\left\langle P_{90}\right\rangle=\frac{E}{\eta_{2}}, \quad\left\langle P_{+}\right\rangle=\frac{E}{\eta}+P_{R} .
$$

In this case, $\left\langle P_{-}\right\rangle,\left\langle P_{90}\right\rangle$ and $\left\langle P_{+}\right\rangle$are the location of the minima of the Gibbs energy.

The time evolution of the dipole phase fractions are governed by the first order ODE system

$$
\left(\begin{array}{c}
\dot{x_{-}} \\
x_{90} \\
x_{+}
\end{array}\right)=\left(\begin{array}{ccc}
-p_{-} & p_{90-} & 0 \\
p_{-} & -\left(p_{90-}+p_{90+}\right) & p_{+} \\
0 & p_{90+} & -p_{+}
\end{array}\right)\left(\begin{array}{c}
x_{-} \\
x_{90} \\
x_{+}
\end{array}\right) .
$$

The system results from the assumption that transitions between the three allowed states occur only to the nearest neighbor. The likelihood to switch out of the $P_{-}$orientation into the $P_{90}$ orientation is denoted by $p_{-}$and the notation for the remaining likelihoods is summarized in Table 2 .

The likelihood $p_{-}$is calculated by

$$
p_{-}=\frac{1}{\tau} \frac{e^{-\gamma G\left(-P_{I}\right)}}{\int_{-\infty}^{-P_{I}} e^{-\gamma G(P)} d P}
$$

where the relaxation time $\tau$ is the reciprocal of the frequency at which dipoles attempt a switch. The likelihood of switching out of the $P_{90}$ orientation into the $P_{-}$orientation is specified by $p_{90-}$ and is calculated by

$$
p_{90-}=\frac{1}{\tau} \frac{e^{-\gamma G\left(-P_{90}\right)}}{\int_{-P_{90}}^{P_{90}} e^{-\gamma G(P)} d P} .
$$

The likelihoods $p_{90+}$ and $p_{+}$are obtained in a similar manner. The likelihoods can also be evaluated in terms of error functions as detailed in Appendix B resulting in,

$$
p_{+}=\frac{1}{\tau} \frac{e^{-\gamma G\left(P_{I}\right)}}{\int_{P_{I}}^{\infty} e^{-\gamma G(P)} d P}=\frac{1}{\tau} \frac{e^{-\alpha\left(P_{I}+b / 2\right)^{2}}}{\frac{1}{2} \sqrt{\frac{\pi}{\alpha}} \cdot \operatorname{erfc}\left(\sqrt{\alpha}\left(P_{I}+b / 2\right)\right)}
$$

where $\alpha=\frac{\gamma \eta}{2}$ and $b=-2 \frac{\left(P_{R}-E\right)}{\eta}$. The likelihoods $p_{90+}, p_{90-}$ and $p_{-}$follow similarly. 


\begin{tabular}{c|c} 
State Transition & notation \\
\hline$P_{-} \rightarrow P_{90}$ & $p_{-}$ \\
$P_{90} \rightarrow P_{-}$ & $p_{90-}$ \\
$P_{90} \rightarrow P_{+}$ & $p_{90+}$ \\
$P_{+} \rightarrow P_{90}$ & $p_{+}$ \\
\hline
\end{tabular}

Table 2: 1-D transition likelihoods.

\section{$5 \quad$ Macroscopic Polarization Model}

Nonuniformities in the lattice structure due to polycrystallinity, material nonhomogeneities and variations across grain boundaries produce a distribution of Helmholtz and Gibbs energy profiles which can be manifested as variations in the local coercive field and local remanent polarization. Other variations can be produced by stress nonhomogeneities and variable effective fields.

To incorporate these effects on route to constructing a macroscopic model, we consider the coercive field $E_{c}$ to be a manifestation of an underlying density $\nu_{1}\left(E_{c}\right)$ rather than fixed values which is typically assumed for single crystals having a uniform lattice structure. To create a macroscopic model for the polarization, we also consider the variation of effective fields in the material. As detailed in [24, 31], an applied field $E$ in a ferroelectric material is augmented by an interaction field $E_{I}$ generated by neighboring dipoles which produce nonhomogeneous effective fields in the material. This, along with various other processes, produces variations in the applied field that can significantly alter the resulting polarization. To incorporate these variations, we consider the effective field $E_{e}=E+E_{I}$ to be distributed about the applied field $E$ with an underlying density for the interaction field $E_{I}$ which we denote by $\nu_{2}\left(E_{I}\right)$. The introduction of variations in the effective field produces domain switching in advance of the remanence point in accordance with observations from experimental data.

The complete macroscopic polarization model for nonhomogeneous, polycrystalline materials with distributed coercive and effective fields is

$$
\mathbf{P}(\mathbf{E})=\int_{0}^{\infty} \int_{-\infty}^{\infty}\langle\mathbf{P}\rangle\left(E ; E_{I}, E_{c}\right) \nu_{1}\left(E_{c}\right) \nu_{2}\left(E_{I}\right) d E_{I} d E_{c}
$$

where $\langle\mathbf{P}\rangle$ is the local polarization kernel given by (6),(12),(19) or (25) when thermal effects are negligible or by (16) or (29) when incorporating thermal relaxation.

\section{$6 \quad$ Model Validation and Properties}

In this section, the ferroelastic models presented in Sections 4.2 and 4.3 are compared with experimental PLZT data reported in [13]. Both ferroelastic switching models employ the thermal activation models presented in Sections 4.1.3 and 4.3.3 to evolve the polarization in time. However, since the measured values exhibit minimal relaxation, both the 1-D and 2-D switching models were computed with a large $\gamma$ and a small $\tau$ which resulted in negligable thermal activation. As reported in [13], the PLZT composition is also very near the rhombohedral-tetragonal morphotropic boundary. To model this nonhomogenous composition, we utilize the macroscopic polarization model of Section 5 .

For the macroscopic models, we make the a priori assumption that the density for the coercieve field is given by

$$
\nu_{1}\left(E_{c}(\sigma)\right)=\left\{\begin{array}{cc}
c_{1} e^{-\bar{E}_{c}^{2}(\sigma) / b_{1}} & e^{-\bar{E}_{c}^{2}(\sigma) / b_{1}}>0 \\
0 & e^{-\bar{E}_{c}^{2}(\sigma) / b_{1}} \leq 0
\end{array}\right.
$$


and the density for the interaction field is given by

$$
\nu_{2}\left(E_{I}\right)=c_{2} e^{-E_{I}^{2} / b_{2}}
$$

both are plotted in Figure 8. The relation $\bar{E}_{c}(\sigma)$ is specified by the single crystal values $(20)$ and $(26)$ and the variance of the coercive field was estimated from the variance in the experimentally measured values in Table 3 . The variance of the effective field was estimated by the degree to which switching occurs before remanence in the PLZT data. The parameters of the densities $\nu_{1}\left(E_{c}\right)$ and $\nu_{2}\left(E_{I}\right)$ used in the model were $c_{1}=27.0, b_{1}=6.5 \times 10^{-4}(\mathrm{MV} / \mathrm{m})^{2}, c_{2}=6.0$ and $b_{2}=5.0 \times 10^{-3}(\mathrm{MV} / \mathrm{m})^{2}$. To implement the macroscopic polarization given by (30), a composite Gaussian quadrature was employed.

We note that additional accuracy can be obtained if general densities $\nu_{1}$ and $\nu_{2}$ are identified in the manner detailed in [27] for $180^{\circ}$ field-induced switching. Extension of the framework in this direction is under present investigation.

The ferroelastic models were programmed in MATLAB and run on a $1.7 \mathrm{Ghz}$ laptop with $512 \mathrm{MB}$ of RAM. The average computational times for each model are given in Table 5. The 2-D ferroelastic model requires the values of the four minima and the saddle points of the Gibbs energy. These nonlinear problems were solved using Newton's method with a line search. The computational cost of the 2-D model includes eight nonlinear solves per time step and $1 \mathrm{LU}$ factorization for the backward Euler time discretization of the thermal activation models. The 1-D ferroelastic single-crystal model does not require the nonlinear Newton solutions which significantly decreases the computational effort. It is also noted that when operating in the absence of thermal activation, the local minimization models given by (19) and (25) are more computationally efficient than the thermal evolution models and should be used when appropriate.

\subsection{2-D Piecewise Ferroelastic Model}

The ferroelectric hysteron or kernel (12) is the starting point for choosing several of the ferroelastic model parameters. The slope of the hysteron in the $180^{\circ}$ switching regime is given by $\eta_{3}^{-1}$ and the slope of the hysteron in the $90^{\circ}$ regime is given by $\eta_{1}^{-1}$. The remanent polarizations in the 1 and 3 direction are defined by the parameters $P_{1 R}$ and $P_{3 R}$ which may have different values depending upon the internal structure of the material. The inflection point $P_{3 I}$ is chosen to determine the coercive field via relation (13). The remaining coefficients $a_{3}, q_{3}$ and $Y_{3}$ affect the slope and intercept of the linear

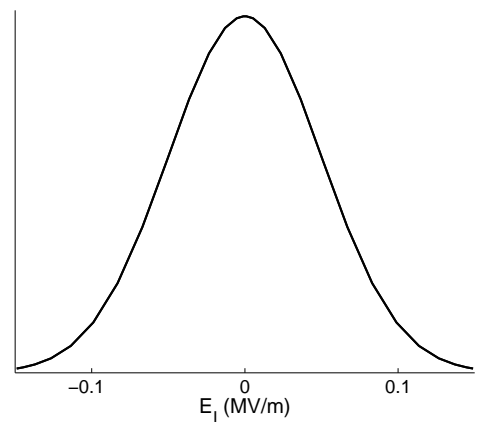

(a)

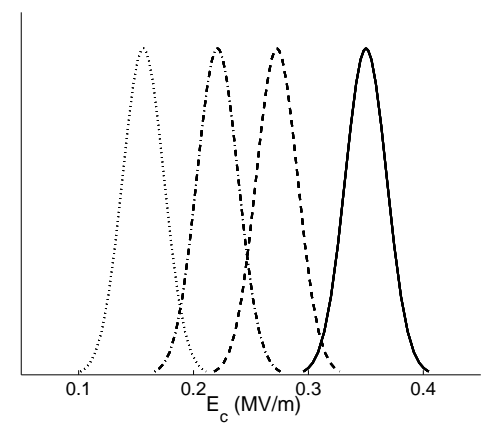

(b)

Figure 8: Typical Macroscopic polarization model densities $\nu_{2}\left(E_{I}\right)$ and $\nu_{1}\left(E_{c}\right)$. (a) Normally distributed interaction field $\nu_{2}\left(E_{I}\right)$ and (b) normally distributed coercive field $\nu_{1}\left(E_{c}\right)$ for varying applied stresses. Here $E_{c}(0)=0.35, E_{c}(-6)=0.27, E_{c}(-10)=0.22$, and $E_{c}(-15)=0.15$. 


\begin{tabular}{c|c|c|c}
$\sigma_{3}(\mathrm{MPa})$ & $P_{3 R}\left(\mathrm{C} / \mathrm{m}^{2}\right)$ & $E_{c}(\mathrm{MV} / \mathrm{m})$ & $E_{c}^{90}(\mathrm{MV} / \mathrm{m})$ \\
\hline 0 & 0.247 & $0.35 \pm 0.01$ & $0.35 \pm 0.01$ \\
-6 & 0.247 & $0.26 \pm 0.01$ & $0.35 \pm 0.01$ \\
-10 & 0.235 & $0.19 \pm 0.02$ & $0.35 \pm 0.02$ \\
-15 & 0.215 & $0.15 \pm 0.02$ & $0.39 \pm 0.02$ \\
\hline
\end{tabular}

Table 3: Experimental values for $\sigma_{3}, P_{3 R}, E_{c}^{90}$, and $E_{c}$ for rhombohedral PLZT from data reported in [13].

$E_{c}(\sigma)$ relation $(20)$. These values can be ascertained by fitting the $E_{c}(\sigma)$ relation to experimental data as illustrated in Figure 9. Finally, $P_{1 I}$ is chosen so that the $90^{\circ}$ switching occurs appropriately as the stress is applied. This can be achieved by plotting the linear $E_{c}^{90}(\sigma)$ relation to ensure that its value is sufficiently large to induce switching at the appropriate stress levels. The values of $q_{13}, q_{31}, a_{13}$ and $a_{31}$ can be chosen from their effect on the $E_{3}-\varepsilon_{3}$ relations. The ferroelastic parameters used to characterize the PLZT data using the 2-D model are summarized in Table 4.

The 2-D model is run with varying applied stresses in the 3 direction as well as an oscillating electric field also in the 3 direction. While the 2-D model is able to employ stresses and fields in the 1 direction, they are set to zero to match experimental conditions. The reader is referred to [13] for details regarding the experimental procedures. The behavior predicted by the 2-D model (30), employing the density choices (31) and (32) and with negligible thermal relaxation is compared to the PLZT data in Figures 10 - 12. It is noted that a possible source of error in the model fit may arise from the rhombohedral nature of the PLZT data since the ferroelastic model is derived in the tetragonal phase. The model parameters were chosen to optimize the fit of the $E_{3}-P_{3}$ and $E_{3}-\varepsilon_{3}$ data shown in Figures 10 and 11. The ferroelastic model characterizes the $90^{\circ}$ switching that occurs in the $E_{3}-P_{3}$ data as a compressive stress is applied to the PLZT sample. As noted in Figure 11, the model also predicts a negative strain due to an applied compressive stress as well as the butterfly nature of the $E_{3}-\varepsilon_{3}$ data. The datafit of the $\sigma_{3}-\varepsilon_{3}$ relation shown in Figure 12 can be optimized by setting the Young's modulus parameters to match the slope of the appropriate part of the $\sigma_{3}-\varepsilon_{3}$ curve. However, this results in an underprediction of the strains in the $E_{3}-\varepsilon_{3}$ curve. Simultaneously optimizing both the $\sigma_{3}-\varepsilon_{3}$ and $E_{3}-\varepsilon_{3}$ relations may be accomplished by using higher-order terms in the electromechanical coupling energy given by (1).

The PLZT data in [13] illustrates the stress-dependence of the remanence and saturation polarization. For applied compressive stresses greater than $15 \mathrm{MPa}$, the remanence and saturation polarization

\begin{tabular}{c|c|c|c} 
parameter & value & parameter & value \\
\hline$P_{1 R}$ & $0.22\left(\mathrm{C} / \mathrm{m}^{2}\right)$ & $a_{3}$ & $0.01(\mathrm{MV} / \mathrm{m})$ \\
$P_{1 I}$ & $0.20\left(\mathrm{C} / \mathrm{m}^{2}\right)$ & $a_{31}$ & $0.01(\mathrm{MV} / \mathrm{m})$ \\
$P_{3 R}$ & $0.24\left(\mathrm{C} / \mathrm{m}^{2}\right)$ & $a_{1}$ & $0.01(\mathrm{MV} / \mathrm{m})$ \\
$P_{3 I}$ & $0.239\left(\mathrm{C} / \mathrm{m}^{2}\right)$ & $a_{13}$ & $0.01(\mathrm{MV} / \mathrm{m})$ \\
$\eta_{1}$ & 20.0 & $q_{3}$ & $500.0(\mathrm{MV} / \mathrm{m})^{2}$ \\
$\eta_{3}$ & 20.0 & $q_{31}$ & $-100.0(\mathrm{MV} / \mathrm{m})^{2}$ \\
$c$ & 20.0 & $q_{1}$ & $500.0(\mathrm{MV} / \mathrm{m})^{2}$ \\
$Y_{1}$ & $50000(\mathrm{MPa})$ & $q_{13}$ & $-100.0(\mathrm{MV} / \mathrm{m})^{2}$ \\
$Y_{3}$ & $20000(\mathrm{MPa})$ & & \\
\hline
\end{tabular}

Table 4: Parameters for 2-D piecewise ferroelastic switching model. 


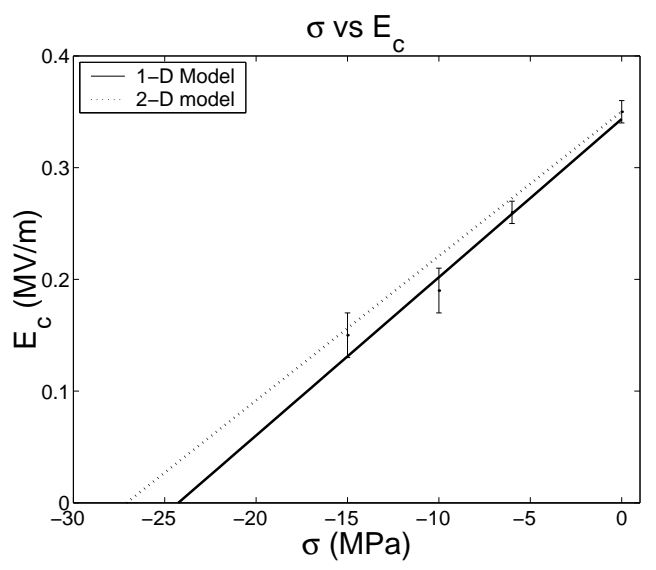

Figure 9: $\sigma_{3}$ vs $E_{c}$ for the 1-D (solid line) and 2-D (dotted line) ferroelastic models where $E_{c}$ is defined by equations $(20)$ or $(26)$.

significantly decrease. Since the remanence polarization is a fixed model parameter, the present formulation of the ferroelastic model should be limited to moderate stress regimes.

\section{$6.2 \quad$ 1-D Piecewise Ferroelastic Model}

The stress-free hysteron or kernel (25) from the ferroelastic model provides the starting point for choosing several of the 1-D ferroelastic model parameters. The slope of the hysteron in the $180^{\circ}$ regime is given by $\eta^{-1}$ and the slope of the hysteron in the $90^{\circ}$ regime is given by $\eta_{2}^{-1}$. The remanent polarization is defined by the parameter $P_{R}$ and the inflection point $P_{I}$ is chosen to determine the coercive field by equation (13). The remaining coefficients $a, q$ and $Y$ affect the slope and intercept of the linear $E_{c}(\sigma)$ relation $(26)$. These values can be ascertained by fitting the $E_{c}(\sigma)$ relation to experimental data as illustrated in Figure 9. Finally, $P_{90 I}$ is chosen so that the $90^{\circ}$ switching occurs appropriately as the stress is applied. This can be done by plotting the linear $E_{c}^{90}(\sigma)$ relation to ensure that its value is large enough to begin switching at the appropriate stress levels. The values of $P_{m}, \eta_{1}, \beta$ and $\Delta$ are defined in equation (22). The parameters used to model the PLZT data are compiled in Table 6 .

The 1-D ferroelastic model is compared to the PLZT data in Figures 13 - 15. The model parameters were chosen to optimize the fit of the $E-P$ and $E-\varepsilon$ data shown in Figures 13 and 14 . As with the 2-D model, the 1-D ferroelastic model characterizes the $90^{\circ}$ switching that occurs in the $E-P$ data as a compressive stress is applied to the PLZT sample, but with less computational cost than the 2-D model. The model also predicts a negative strain due to an applied compressive stress as well as the butterfly nature of the $E-\varepsilon$ data.

We note that for this characterization regime, the simplified 1-D model provides approximately the same accuracy as the 2-D model but is nearly 2 orders of magnitude faster as indicated in Table 5.

\begin{tabular}{c|c|c} 
& Single Crystal & Macroscopic \\
\hline 1-D & $0.3 \mathrm{sec}$ & $67.8 \mathrm{sec}$ \\
$2-\mathrm{D}$ & $4.6 \mathrm{sec}$ & $1155.4 \mathrm{sec}$ \\
\hline
\end{tabular}

Table 5: Average computational times for the ferroelastic switching models running on a 1.7Ghz laptop with $512 \mathrm{MB}$ of RAM. The applied electric field was $E=E_{0} \sin (3 \pi t)$ where $\mathrm{t}=[0,1]$ with $\Delta t=0.005$. 


\begin{tabular}{c|c|c|c} 
parameter & value & parameter & value \\
\hline$P_{R}$ & $0.24\left(\mathrm{C} / \mathrm{m}^{2}\right)$ & $a$ & $0.01(\mathrm{MV} / \mathrm{m})$ \\
$P_{I}$ & $0.239\left(\mathrm{C} / \mathrm{m}^{2}\right)$ & $q$ & $500.0(\mathrm{MV} / \mathrm{m})^{2}$ \\
$P_{90 I}$ & $0.015\left(\mathrm{C} / \mathrm{m}^{2}\right)$ & $Y$ & $20000(\mathrm{MPa})$ \\
$\eta$ & 20.0 & $\eta_{2}$ & 20.0 \\
\hline
\end{tabular}

Table 6: Parameters for the 1-D piecewise ferroelastic switching model.

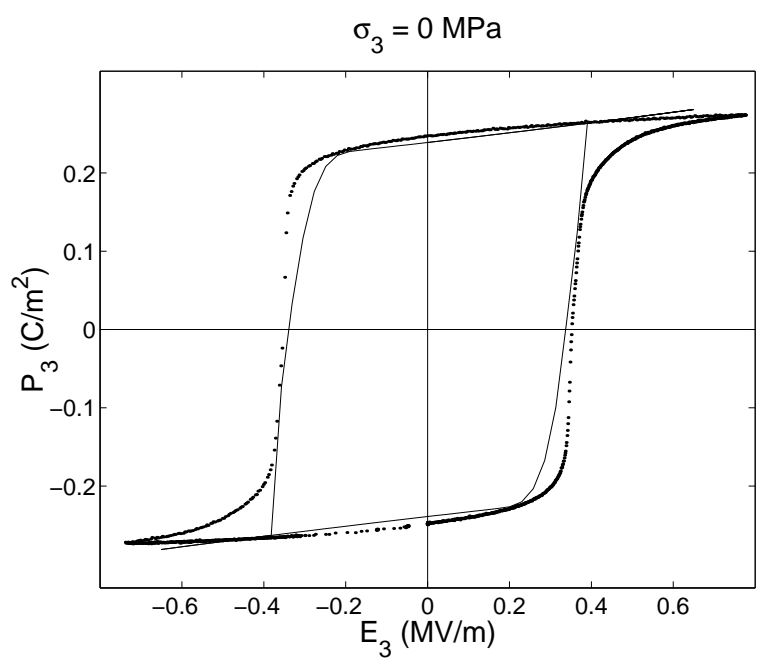

(a)

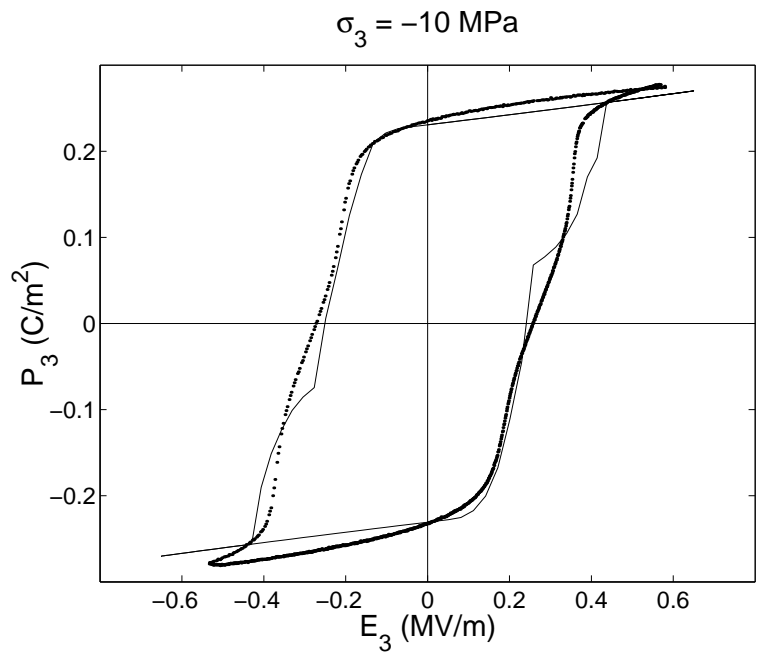

(c)



(b)

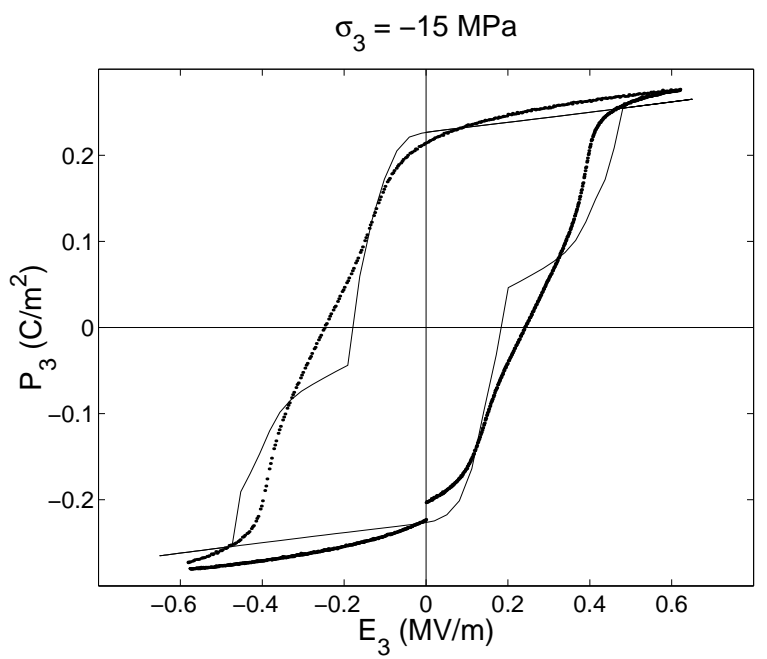

(d)

Figure 10: Macroscopic $E_{3}-P_{3}$ relation for varying applied stresses. Homogenized 2-D ferroelastic model (-) and experimental PLZT data ( $\cdots$ ): (a) $\sigma_{3}=0 \mathrm{MPa}$, (b) $\sigma_{3}=-6 \mathrm{MPa}$, (c) $\sigma_{3}=-10$ $\mathrm{MPa}$, and (d) $\sigma_{3}=-15 \mathrm{MPa}$. 


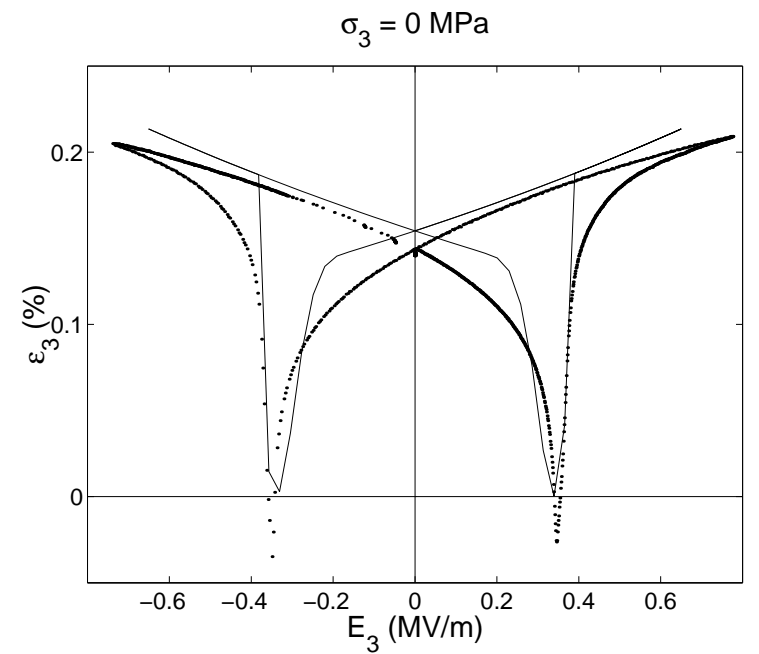

(a)

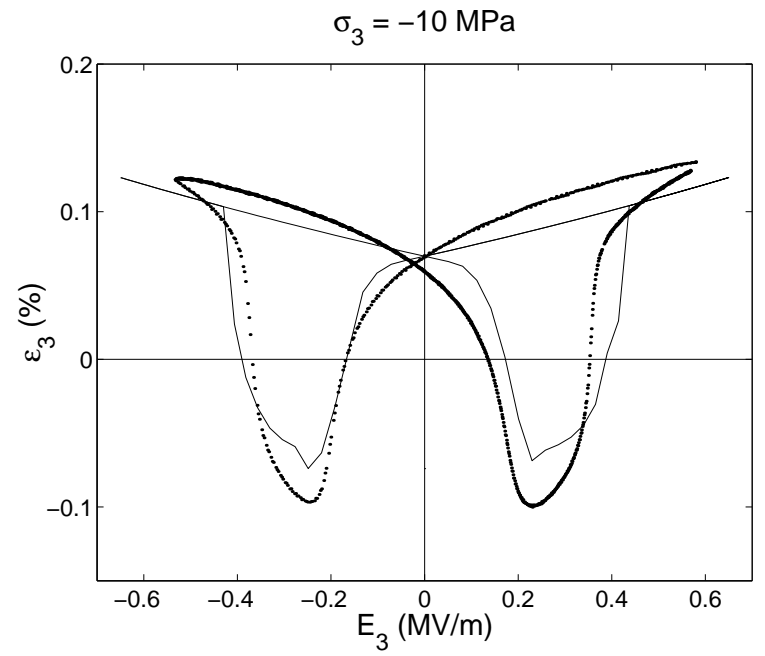

(c)

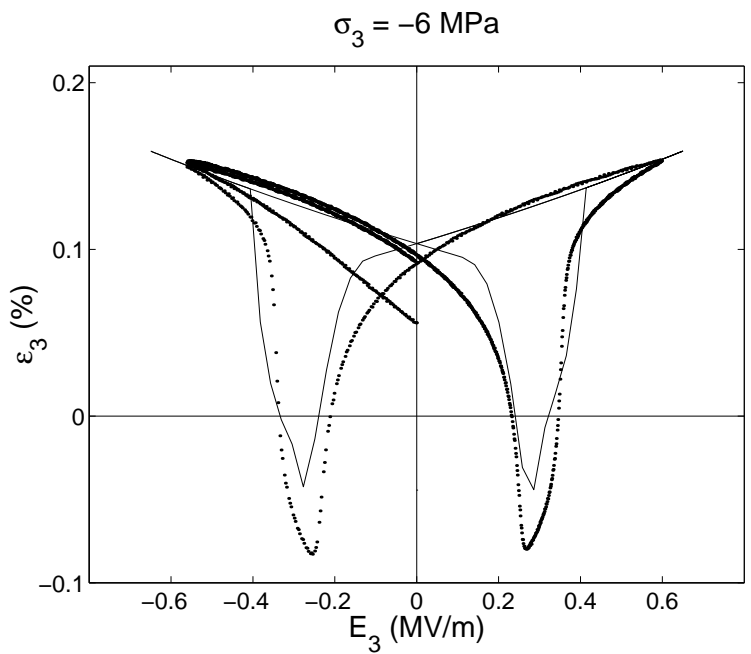

(b)

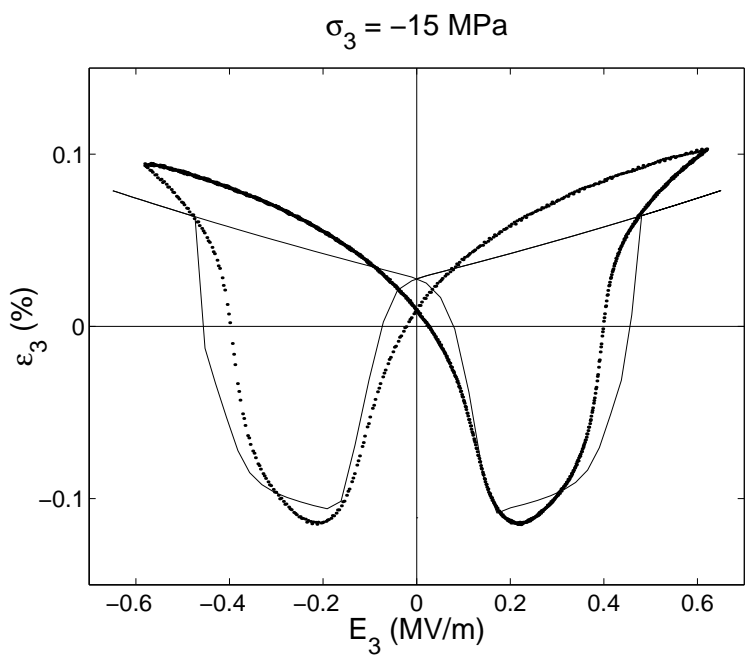

(d)

Figure 11: Macroscopic $E_{3}-\varepsilon_{3}$ relation for varying applied stresses. Homogenized 2-D ferroelastic model (- $)$ and experimental PLZT data $(\cdots)$ : (a) $\sigma_{3}=0 \mathrm{MPa}$, (b) $\sigma_{3}=-6 \mathrm{MPa}$, (c) $\sigma_{3}=-10$ $\mathrm{MPa}$, and (d) $\sigma_{3}=-15 \mathrm{MPa}$. 


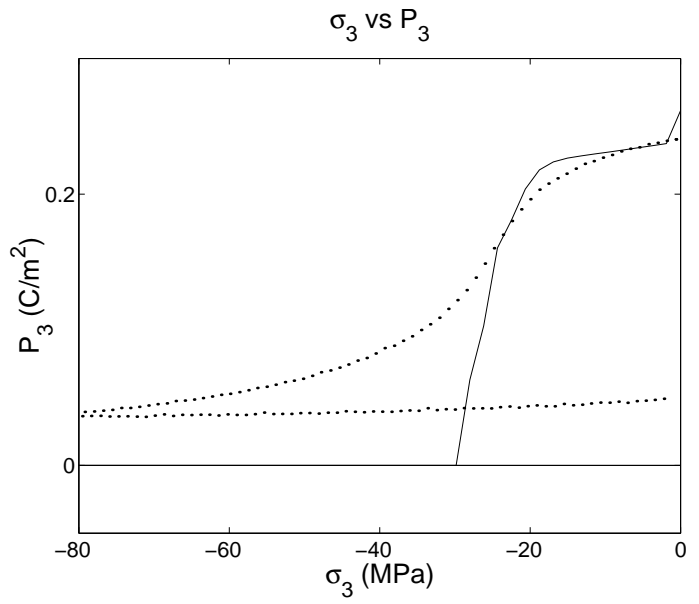

(a)

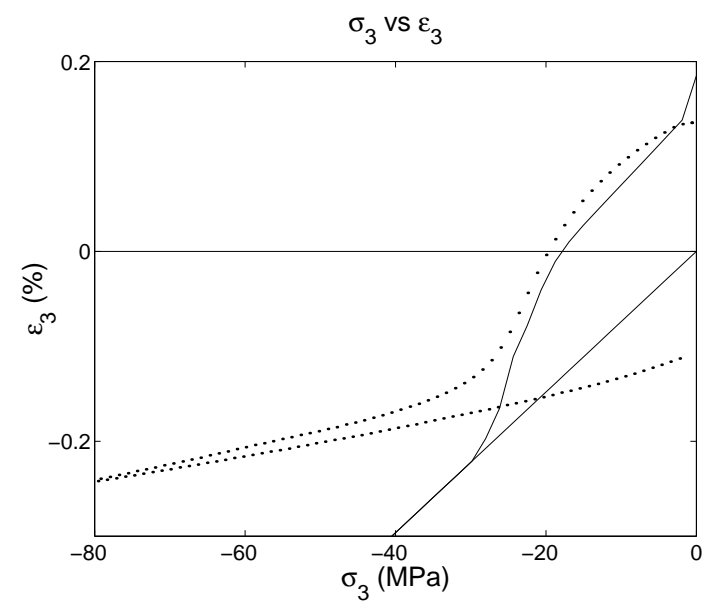

(b)

Figure 12: (a) Macroscopic $\sigma_{3}-P_{3}$ relation and (b) macroscopic $\sigma_{3}-\varepsilon_{3}$ relation. Homogenized 2-D ferroelastic model $(-)$ and experimental PLZT data $(\cdots)$.

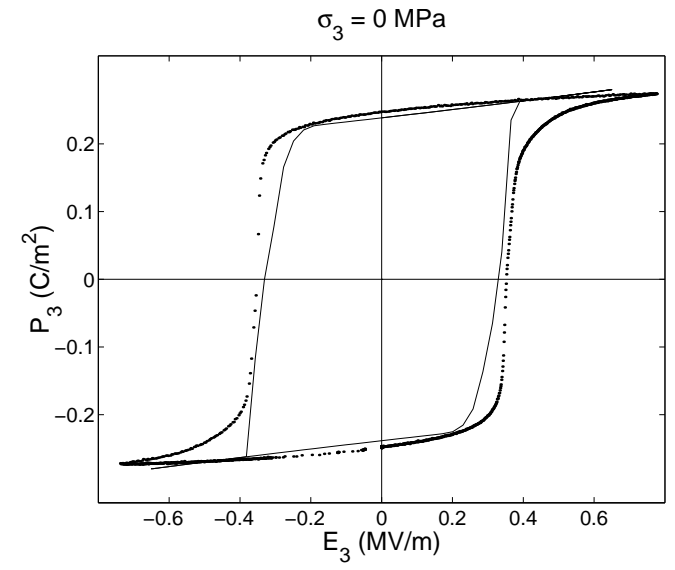

(a)

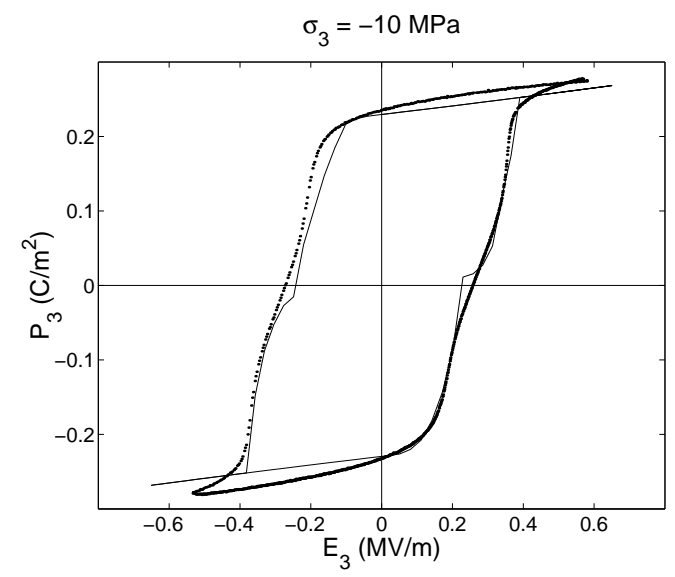

(c)

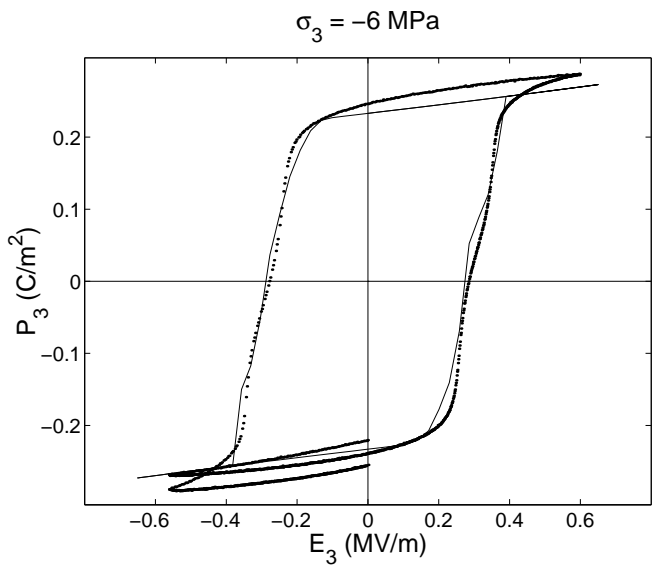

(b)

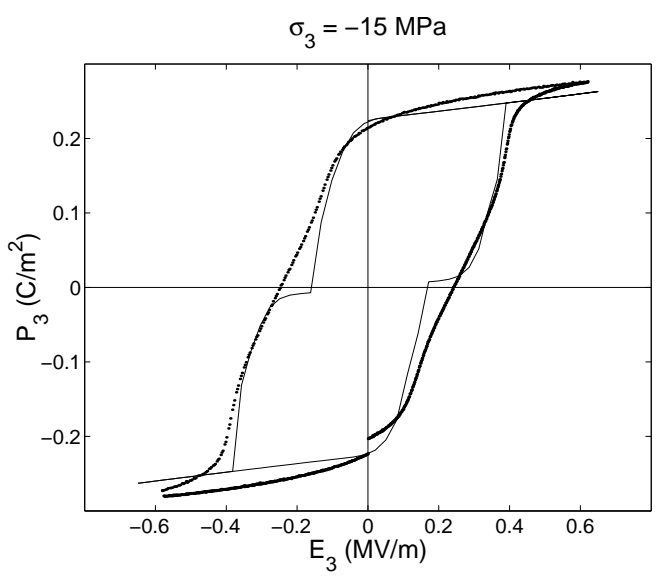

(d)

Figure 13: Macroscopic $E-P$ relation for varying applied stresses. Homogenized 1-D ferroelastic model (- and experimental PLZT data $(\cdots):$ (a) $\sigma_{3}=0 \mathrm{MPa}$, (b) $\sigma_{3}=-6 \mathrm{MPa}$, (c) $\sigma_{3}=-10$ $\mathrm{MPa}$, and (d) $\sigma_{3}=-15 \mathrm{MPa}$. 


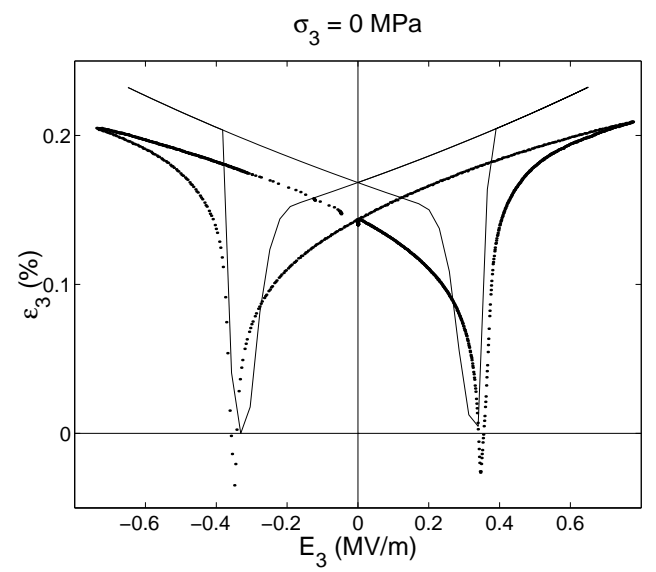

(a)

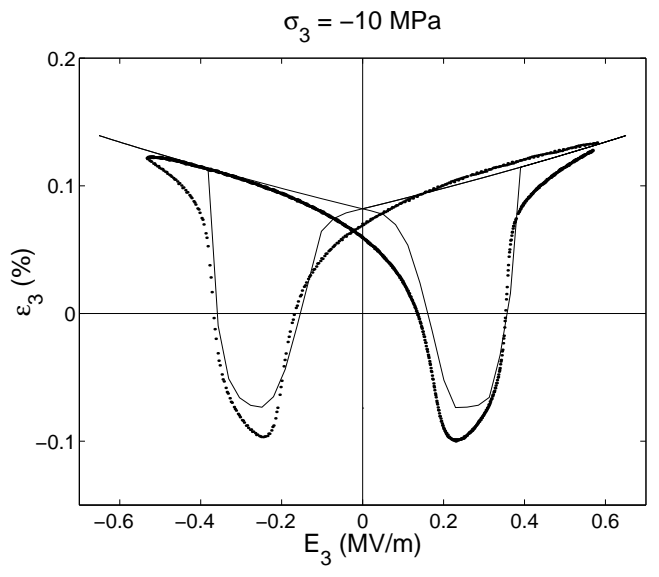

(c)

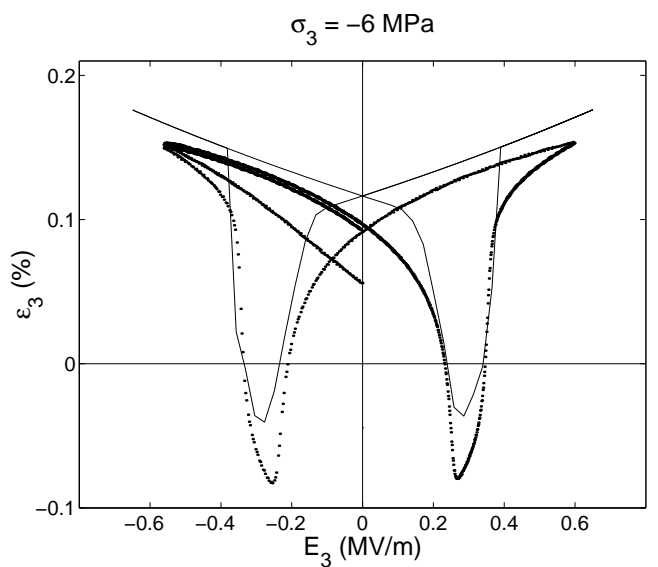

(b)

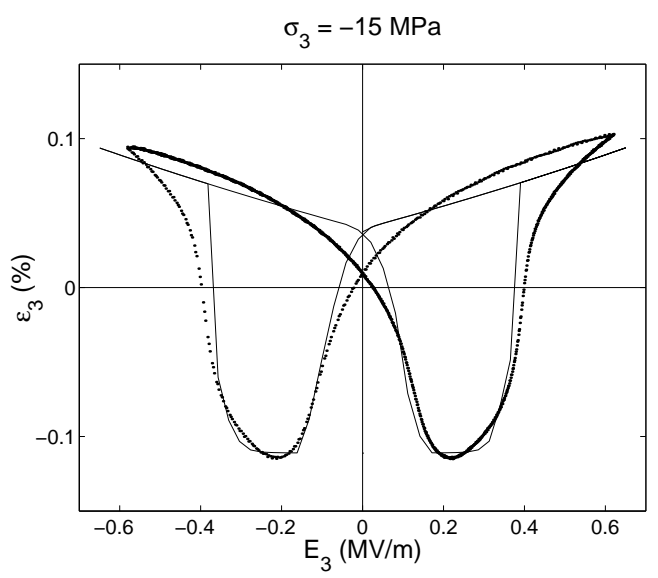

(d)

Figure 14: Macroscopic $E-\varepsilon$ relation for varying applied stresses. Homogenized 1-D ferroelastic model (- and experimental PLZT data ( $\cdots$ ): (a) $\sigma_{3}=0 \mathrm{MPa}$, (b) $\sigma_{3}=-6 \mathrm{MPa}$, (c) $\sigma_{3}=-10$ $\mathrm{MPa}$, and (d) $\sigma_{3}=-15 \mathrm{MPa}$.

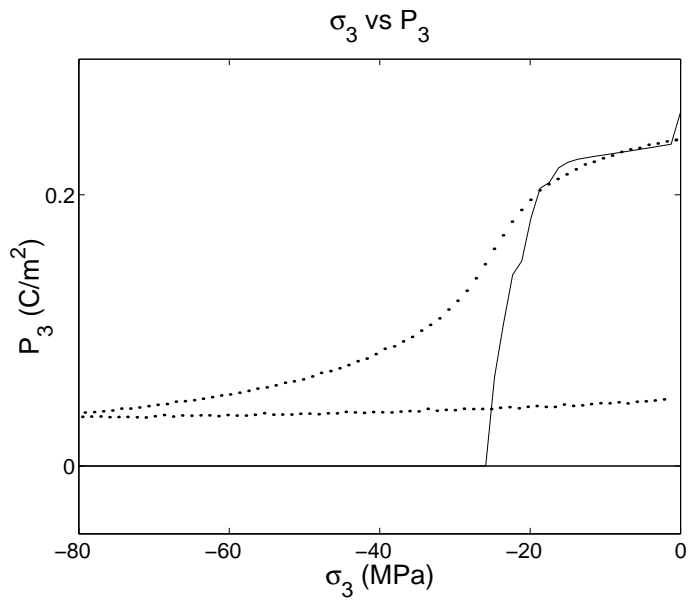

(a)

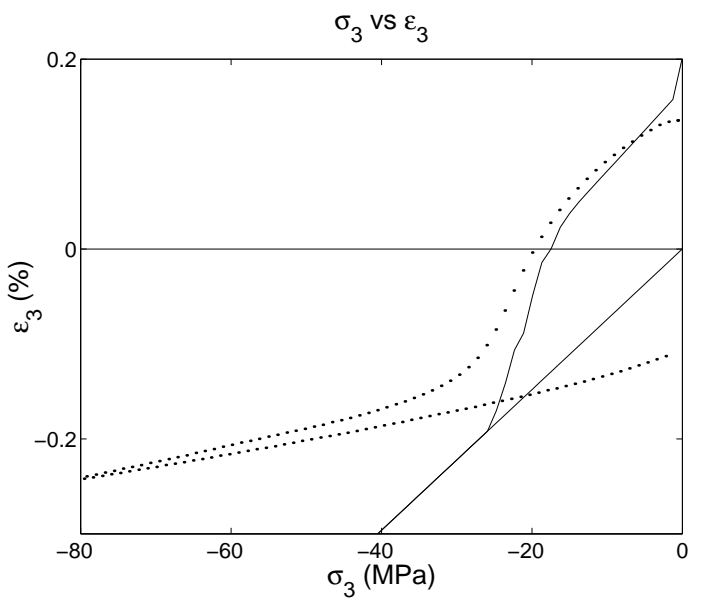

(b)

Figure 15: (a) Macroscopic $\sigma-P$ relation and (b) macroscopic $\sigma-\varepsilon$ relation. Homogenized 1-D ferroelastic model $(-)$ and experimental PLZT data $(\cdots)$. 


\section{$7 \quad$ Concluding Remarks}

This paper addresses the development of a framework for characterizing stress-induced $90^{\circ}$ and $180^{\circ}$ switching inherent to ferroelectric materials in a manner suitable for subsequent transducer and control design. The model builds upon the homogenized energy framework developed for ferroelectric materials in [31] and extended to provide a unified characterization framework for ferroic compounds in [29, 30].

In the first step of the development, three techniques are employed to construct Helmholtz and Gibbs energy functionals used to characterize the electromechanical behavior of homogeneous, single crystal compounds. The first exploits a classical Landau-Devonshire energy formulation whereas the second employs a piecewise quadratic formulation to eliminate numerical and physical restrictions inherent to high-order polynomials comprising the Landau-Devonshire functional. Both choices quantify the 2-D polarization $\mathbf{P}$ as a function of $2-\mathrm{D}$ stress and field inputs. The third choice is similar to that employed for SMA [14, 23, 24, 29] in the sense that it is 1-D with three wells corresponding to $\pm 180^{\circ}$ and $90^{\circ}$ equilibria. The construction of this functional is phenomenological but the resulting decrease in dimension significantly diminishes implementation time. In all three cases, the functionals are directly minimized to provide kernels for characterization in the absence of thermal relaxation or balanced with the relative thermal energy through Boltzmann principles to incorporate relaxation phenomena.

In the second step of the development, the effects of material nonhomogeneities, polycrystallinity, and variable effective fields are incorporated by assuming that properties such as local coercive and interaction fields are manifestations of underlying distributions rather than constants. Stochastic homogenization in this manner provides a macroscopic model which characterizes field and stressinduced $90^{\circ}$ and $180^{\circ}$ switching in ferroelectric materials.

In the present formulation, a priori choices of normal densities are made when quantifying local coercive and interaction field distributions. This yields macroscopic models with a small number of parameters to be identified but restricts the accuracy of the framework to realizations of the $a$ priori choices. Alternatively, one can employ general densities to be identified through least squares techniques in the manner detailed for field-induced $180^{\circ}$ ferroelectric switching in [27]. This greatly increases the flexibility and accuracy of the model while maintaining the same complexity with regard to subsequent control design. Extension of the framework in this direction is under present investigation.

One way in which the homogenized energy framework has been employed for control design is through the construction of inverse filters which can be used to linearize the transducer response. Details illustrating this technique in the context of the magnetic model quantifying $180^{\circ}$ field-induced switching can be found in [20] and development of inverse filters for the present theory constitute a second facet of current research.

\section{Appendix A: Coefficients of Stress Dependent Gibbs Energy}

We summare here the coefficients employed in the energy relations of Section 4.2.1. The coefficients result from substituting the equations for the strains into the Gibbs energy relation and results in the following: 


$$
\begin{aligned}
\gamma_{1} & =-\frac{q_{31}^{2}}{2 Y_{3}}-\frac{q_{1}^{2}}{2 Y_{1}} \\
\gamma_{2} & =-\frac{q_{13}^{2}}{2 Y_{1}}-\frac{q_{3}^{2}}{2 Y_{3}} \\
\gamma_{3} & =c-\frac{q_{3} q_{31}}{Y_{3}}-\frac{q_{13} q_{1}}{Y_{1}} \\
\gamma_{4} & =-\frac{a_{31} q_{31}}{Y_{3}}-\frac{a_{1} q_{1}}{Y_{1}} \\
\gamma_{5} & =-\frac{q_{13} a_{13}}{Y_{1}}-\frac{a_{3} q_{3}}{Y_{3}} \\
\gamma_{6} & =-\frac{a_{3} q_{31}}{Y_{3}}-\frac{a_{13} q_{1}}{Y_{1}} \\
\gamma_{7} & =-\frac{a_{31} q_{3}}{Y_{3}}-\frac{a_{1} q_{13}}{Y_{1}} \\
\gamma_{8} & =-\frac{a_{31}^{2}}{2 Y_{3}}-\frac{a_{1}^{2}}{2 Y_{1}}+\frac{\eta_{1}}{2 P_{1 I}}\left(P_{1 I}-P_{1 R}\right)-\frac{q_{31}}{Y_{3}} \sigma_{3} \\
\gamma_{9} & =-\frac{a_{13}^{2}}{2 Y_{1}}-\frac{a_{3}^{2}}{2 Y_{3}}+\frac{\eta_{3}}{2}-\frac{q 3}{Y_{3}} \sigma_{3} \\
\gamma_{10} & =-\frac{a_{1} a_{13}}{Y_{1}}-\frac{a_{31} a_{3}}{Y_{3}} \\
\gamma_{11} & =-E_{1}-\frac{\sigma_{3} a_{31}}{Y_{3}} \\
\gamma_{12} & =-E_{3}-\eta_{3} P_{3 R}-\frac{\sigma_{3} a_{3}}{Y_{3}} \\
\gamma_{13} & =-\frac{\eta_{1}}{2}\left(P_{1 I}-P_{1 R}\right) P_{1 R}-\frac{\sigma_{3}^{2}}{2 Y 3}+\frac{\eta_{3} P_{3 r}^{2}}{2} \\
\gamma_{3} & =1
\end{aligned}
$$

\section{Appendix B: Conversion of Polarization Integrals to Error Functions}

For fixed temperatures, we employ the piecewise quadradic relation Helmholtz energy

$$
\psi(P)= \begin{cases}\frac{1}{2} \eta\left(P+P_{R}\right)^{2} & , \quad P \leq-P_{I} \\ \frac{1}{2} \eta\left(P-P_{R}\right)^{2} & , \quad P \geq P_{I} \\ \frac{1}{2} \eta\left(P_{I}-P_{R}\right)\left(\frac{P^{2}}{P_{I}}-P_{R}\right) & , \quad|P|<P_{I}\end{cases}
$$

where $P_{I}$ and $P_{R}$ denote the positive inflection point and polarization at which the minimum occurs. The resulting Gibbs energy is derived by combining the potential energy of a dipole in the field with the Helmholtz energy throughout the lattice to yield

$$
G(P, E)=\psi(P)-E P .
$$

In the thermal evolution model, integrals of the form

$$
\int_{P_{I}}^{\infty} e^{-\gamma G(P, E)} d P \quad \text { and } \quad \int_{P_{I}}^{\infty} P e^{-\gamma G(P, E)} d P
$$

must be computed where $\gamma=\sqrt{\frac{V}{K T}}$. Since $\psi$ is a piecewise quadradic, the integrals are Gaussians 
and can be represented as error functions for computational efficiency. It is helpful to note that

$$
\int_{P_{I}}^{\infty} e^{-\alpha\left(P-P_{R}\right)^{2}} d P=\int_{P_{I}-P_{R}}^{\infty} e^{-\alpha z^{2}} d z=\frac{1}{2} \sqrt{\frac{\pi}{\alpha}} \cdot \operatorname{erfc}\left(\sqrt{\alpha}\left(P_{I}-P_{R}\right)\right) .
$$

The first integral of interest is

$$
\begin{gathered}
\int_{P_{I}}^{\infty} e^{-\gamma G(P, E)} d P=\int_{P_{I}}^{\infty} e^{-\gamma\left(\frac{\eta}{2}\left(P-P_{R}\right)^{2}-E P\right)} d P=\int_{P_{I}}^{\infty} e^{-\frac{\gamma \eta}{2}\left(\left(P-P_{R}\right)^{2}-\frac{2}{\eta} E P\right)} d P \\
=\int_{P_{I}}^{\infty} e^{-\frac{\gamma \eta}{2}\left(P^{2}+\left(-2 P_{R}-\frac{2}{\eta} E\right) P+P_{R}^{2}\right)} d P=\int_{P_{I}}^{\infty} e^{-\alpha\left(P^{2}+b P+P_{R}^{2}\right)} d P
\end{gathered}
$$

where $\alpha=\frac{\gamma \eta}{2}$ and $b=-2\left(P_{R}-\frac{E}{\eta}\right)$. Completing the square results in

$$
\int_{P_{I}}^{\infty} e^{-\alpha\left(P^{2}+b P+P_{R}^{2}\right)} d P=\int_{P_{I}}^{\infty} e^{-\alpha\left(\left(P+\frac{b}{2}\right)^{2}+P_{R}^{2}-\frac{b^{2}}{4}\right)} d P=\int_{P_{I}}^{\infty} e^{-\alpha\left(\left(P+\frac{b}{2}\right)^{2}+\beta\right)} d P
$$

where $\beta=\gamma \eta\left(\frac{b^{2}}{4}-\frac{P_{R}^{2}}{2}\right)$. This yields

$$
\int_{P_{I}}^{\infty} e^{-\alpha\left(\left(P+\frac{b}{2}\right)^{2}+\beta\right)} d P=e^{\beta} \int_{P_{I}}^{\infty} e^{-\alpha\left(\left(P+\frac{b}{2}\right)^{2}\right)} d P=e^{\beta} \frac{1}{2} \sqrt{\frac{\pi}{\alpha}} \cdot \operatorname{erfc}\left(\sqrt{\alpha}\left(P_{I}+\frac{b}{2}\right)\right)
$$

which gives

$$
\int_{P_{I}}^{\infty} e^{-\gamma G(P, E)} d P=e^{\beta} \frac{1}{2} \sqrt{\frac{\pi}{\alpha}} \cdot \operatorname{erfc}\left(\sqrt{\alpha}\left(P_{I}+\frac{b}{2}\right)\right) .
$$

For the next integral, it is helpful to note that

$$
\int_{P_{I}}^{\infty} P e^{-\alpha P^{2}} d P=\frac{1}{-2 \alpha} \int_{P_{I}-P_{R}}^{\infty}(-2 \alpha P) e^{-\alpha P^{2}} d P=\left.\frac{1}{-2 \alpha} e^{-\alpha P^{2}}\right|_{P i} ^{\infty}=\frac{e^{-\alpha P_{I}^{2}}}{2 \alpha} .
$$

Following the procedure above, we arrive at

$$
\int_{P_{I}}^{\infty} P e^{-\gamma G(P, E)} d P=e^{\beta} \int_{P_{I}}^{\infty} P e^{-\alpha\left(\left(P+\frac{b}{2}\right)^{2}\right)} d P
$$

where $\alpha, \beta$ and $b$ are previously defined. Multiplication by unity followed by addition and substraction of the same expression yields

$$
\begin{gathered}
e^{\beta} \int_{P_{I}}^{\infty} P e^{-\alpha\left(\left(P+\frac{b}{2}\right)^{2}\right)} d P=e^{\beta} \frac{-2 \alpha}{-2 \alpha}\left[\int_{P_{I}}^{\infty}\left(P+\frac{b}{2}\right) e^{-\alpha\left(\left(P+\frac{b}{2}\right)^{2}\right)} d P-\int_{P_{I}}^{\infty} \frac{b}{2} e^{-\alpha\left(\left(P+\frac{b}{2}\right)^{2}\right)} d P\right] \\
=\frac{e^{\beta}}{-2 \alpha}\left[\int_{P_{I}}^{\infty}(-2 \alpha)\left(P+\frac{b}{2}\right) e^{-\alpha\left(\left(P+\frac{b}{2}\right)^{2}\right)} d P+(\alpha b) \int_{P_{I}}^{\infty} e^{-\alpha\left(\left(P+\frac{b}{2}\right)^{2}\right)} d P\right] \\
=\frac{e^{\beta}}{-2 \alpha}\left[\left.e^{-\alpha\left(\left(P+\frac{b}{2}\right)^{2}\right)}\right|_{P i} ^{\infty}+\frac{\alpha b}{2} \sqrt{\frac{\pi}{\alpha}} \cdot \operatorname{erfc}\left(\sqrt{\alpha}\left(P_{I}+\frac{b}{2}\right)\right)\right] \\
=\frac{e^{\beta}}{2 \alpha}\left[e^{-\alpha\left(\left(P_{I}+\frac{b}{2}\right)^{2}\right)}-\frac{\alpha b}{2} \sqrt{\frac{\pi}{\alpha}} \cdot \operatorname{erfc}\left(\sqrt{\alpha}\left(P_{I}+\frac{b}{2}\right)\right)\right] .
\end{gathered}
$$

Thus the final relation is

$$
\int_{P_{I}}^{\infty} P e^{-\gamma G(P, E)} d P=\frac{e^{\beta}}{2 \alpha}\left[e^{-\alpha\left(\left(P_{I}+\frac{b}{2}\right)^{2}\right)}-\frac{\alpha b}{2} \sqrt{\frac{\pi}{\alpha}} \cdot \operatorname{erfc}\left(\sqrt{\alpha}\left(P_{I}+\frac{b}{2}\right)\right)\right] .
$$




\section{Acknowledgements}

The authors sincerely thank Chris Lynch for providing the PLZT data employed in Section 6. The breadth of behavior spanned by this data both motivated mechanisms for expanding the scope of the model and provided a means of validating attributes of the model over a range of input stresses. The research of BLB and RCS was supported through the NSF grant CMS-0099764 and the Air Force Office of Scientific Research under the grants AFOSR-F49620-01-1-0107 and AFOSR-FA9550-04-10203. The work of SS and SJK was supported in part by the National Science Foundation through the grant DMII-0134464.

\section{References}

[1] M. Achenbach and I. Müller, "Simulation of material behavior of alloys with shape memory," Archives of Mechanics, 37(6), pp. 573-585, 1985.

[2] B.L. Ball and R.C. Smith, "A stress-Dependent Hysteresis Model for PZT-Based Transducers," Proceedings of the SPIE, Vol. 5383, pp. 23-30, Smart Structures and Materials, San Diego, CA, 2004.

[3] M. Brokate and J. Sprekels, Hysteresis and Phase Transitions, Springer-Verlag, New-York, 1996.

[4] W. Cao, S. Tavener and S. Xie, "Simulation of boundary condition influence in a second-order ferroelectric phase transition," Journal of Applied Physics, 86(10), pp. 5739-5746, 1999.

[5] R.E. Cohen, Ed., First-Principles Calculations for Ferroelectrics, American Institute of Physics, Woodbury, NY, 1998.

[6] P. Ge and M. Jouaneh, "Modeling hysteresis in piezoceramic actuators," Precision Engineering, 17, pp. 211-221, 1995.

[7] M.J. Haun, E. Furman, S.J. Jang, H.A. McKinstry and L.E. Cross, "Thermodynamic theory of $\mathrm{PbTiO}_{3}, "$ Journal of Applied Physics, 62(8), pp. 3331-3338, 1987.

[8] M. Kamlah and Q. Jiang, "A constitutive model for ferroelectric PZT ceramics under uniaxial loading," Smart Materials and Structures, 8, pp. 441-459, 1999.

[9] S.J. Kim, S. Seelecke, B.L. Ball, and R.C. Smith, "A Rate-Dependent Two-Dimensional Free Energy Model for Ferroelectric Single Crystals," Continuum Mechanics and Thermodynamics, submitted.

[10] C.M. Landis, "Non-linear constitutive modeling of ferroelectrics," Current Opinion in Solid State and Materials Science, 8, pp. 59-69, 2004.

[11] W.F. Li and G.J. Weng, "A theory of ferroelectric hystersis with superimposed stress," Journal of Applied Physics, 91(6), pp. 3806-3815, 2002.

[12] M.E. Lines and A.M. Glass, Principles and Applications of Ferroelectrics and Related Materials, Oxford University Press, Oxford, UK, 1977, Oxford Classics Series, 2001.

[13] C.S. Lynch, "The effect of uniaxial stress on the electro-mechanical response of 8/65/35 PLZT," Acta Materialia, 44(10), pp. 4137-4148, 1996. 
[14] J.E. Massad and R.C. Smith, "A homogenized free energy model for hysteresis in thin-film shape memory alloys," CRSC Technical Report CRSC-TR04-26; International Journal on the Science and Technology of Condensed Matter Films, to appear.

[15] J.E. Massad, R.C. Smith and G.P. Carman, "A Free Energy Model for Thin-Film Shape Memory Alloys," Proceedings of the SPIE, Volume 5049, pp. 13-23, Smart Structures and Materials, San Diego, CA, 2003.

[16] L.D. Mauck and C.S. Lynch, "Thermo-electro-mechanical behavior of ferroelectric materials. Part I: A computational micromechanical model versus experimental results," Journal of Intelligent Material Systems and Structures, 14, pp. 587-602, 2003.

[17] L.D. Mauck and C.S. Lynch, "Thermo-electro-mechanical behavior of ferroelectric materials. Part II: Introduction of rate and self-heating effects," Journal of Intelligent Material Systems and Structures, 14, pp. 605-621, 2003.

[18] K. Mossi, Z. Ounaies, R.C. Smith and B.L. Ball, "Prestressed curved actuators: characterization and modeling of their piezoelectric behavior," Proceedings of the SPIE, Vol. 5053, pp. 423-435, Smart Structures and Materials, San Diego, CA, 2003.

[19] S. Nambu and D. A. Sagala, "Domain formation and elastic long-range interaction in ferroelectric perovskites," Physical Review B, 50(9), pp. 5838-5847, 1994.

[20] J. Nealis and R.C. Smith, "Robust control of magnetostrictive actuator," Proceedings of the SPIE, Volume 5049, pp. 221-232, Smart Structures and Materials, San Diego, CA, 2003.

[21] G. Robert, D. Damjanovic and N. Setter, "Preisach modeling of piezoelectric nonlinearity in ferroelectric ceramics," Journal of Applied Physics, 89(9), pp. 5067-5074, 2001.

[22] S. Seelecke and O. Heintze, "A model for the strain-rate dependent inner loop behavior of NiTi," Preprint.

[23] S. Seelecke and I. Müller, "Shape memory alloy actuators in smart structures — Modeling and simulation," ASME Applied Mechanics Reviews, 57(1), pp. 23-46, 2004.

[24] R.C. Smith, Smart Material Systems: Model Development, SIAM, Philadelophi, PA, 2005.

[25] R.C. Smith and M.J. Dapino, "A homogenized energy theory for ferromagnetic hysteresis," IEEE Transactions on Magnetics, submitted.

[26] R.C. Smith, M.J. Dapino and S. Seelecke, "A free energy model for hysteresis in magnetostrictive transducers," Journal of Applied Physics, 93(1), pp. 458-466, 2003.

[27] R.C. Smith, A. Hatch, B. Mukherjee and S. Liu, "A homogenized energy model for hysteresis in ferroelectric materials: General density formulation," CRSC Technical Report CRSC-TR04-23; Journal of Intelligent Material Systems and Structures, to appear.

[28] R.C. Smith and S. Seelecke, "An energy formulation for Preisach models," Proceedings of the SPIE, Volume 4693, pp. 173-182, Smart Structures and Materials, San Diego, CA, 2002.

[29] R.C. Smith, S. Seelecke, M.J. Dapino and Z. Ounaies, "A unified model for hysteresis in ferroic materials," Proceedings of the SPIE, Volume 5049, pp. 88-99, Smart Structures and Materials, San Diego, CA, 2003. 
[30] R.C. Smith, S. Seelecke, M.J. Dapino and Z. Ounaies, "A unified framework for modeling hysteresis in ferroic materials," CRSC Technical Report CRSC-TR04-35; Journal of the Mechanics and Physics of Solids, submitted.

[31] R.C. Smith, S. Seelecke, Z. Ounaies and J. Smith, "A free energy model for hysteresis in ferroelectric materials," Journal of Intelligent Material Systems and Structures, 14(11), pp. 719-739, 2003.

[32] A.N. Soukhojak and Y.-M. Chiang, "Generalized rheology of active materials," Journal of Applied Physics, 88(11), pp. 6902-6909, 2000.

[33] X. Zhou and A. Chattopadhyay, "Hysteresis behavior and modeling of piezoceramic actuators," Transactions of the ASME, 68, pp. 270-277, 2001.

[34] D. Zwillinger, Editor-in-Chief, CRC Standard Mathematical Tables and Formulae, 30th Edition, CRC Press, Boca Raton, 1996. 